\title{
The mutual specification of genres and audiences: Reflective two-mode centralities in person-to-culture data
}

\begin{abstract}
Recent developments at the intersection of cultural sociology and network theory suggest that the relations between persons and the cultural forms they consume can be productively analyzed using conceptual resources and methods adapted from network analysis. In this paper, I seek to contribute to this developing line of thinking on the culture-networks link as it pertains to the sociology of taste. I present a general analytic and measurement framework useful for rethinking traditional survey (or population) based data on individuals and their cultural choices as a two mode persons $\times$ genres network. The proposed methodological tools allow me to develop a set of "reflective" metrics useful for ranking both persons and genres in terms of the pattern of choices and audience composition embedded in the cultural network. The empirical analysis shows that these metrics have both face and criterion validity, allowing us to extract useful information that would remain out of reach of standard quantitative strategies. I close by outlining the analytic and substantive implications of the approach.
\end{abstract}

Keywords: Genres, Audiences, Centrality, Two Mode Networks, Omnivorousness 


\section{Introduction}

Recent developments at the intersection of cultural sociology and network theory provide novel ways to move beyond traditional conceptualizations of networks as the "conduits" through which cultural contents flow and of culture as a disembodied abstraction devoid of a relational basis (Lizardo, 2006; Vaisey and Lizardo, 2010; Lewis et al., 2012). A key advance in this line of thinking is the importation of network imagery and analytic tools for the analysis of the sort of large-scale quantitative data that has been the bread and butter of the sociology of taste (e.g. Bourdieu, 1984; Peterson and Kern, 1996; Warde et al., 2008). The key is the re-conceptualize the usual records of responses (choice, belief, adoption, positive or negative evaluation, and so on) at the individual level with respect to a given set of cultural forms, as a record of the network of relations obtaining between individuals and the relevant set of cultural objects under investigation. This network can then be productively analyzed using conceptual and methodological resources adapted from network science and network analysis (Lizardo, 2014; Pachucki and Breiger, 2010; Sonnett, 2016). These efforts promise to bring to bear analytic specificity and substantive bite to Peterson's (1983) re-thinking of the expressive consumption of cultural objects on the part of individuals as empirically detectable patterns of cultural choice (see also DiMaggio, 1987).

In this paper I seek to contribute to this developing line of thinking on the culturenetworks link as it pertains to the sociology of taste. I present a general analytic and measurement framework useful for conceptualizing expressive choice patterns as inducing a network of persons connected to the cultural genres they choose. The approach is particularly applicable to work on tastes and cultural choices using a "large-N" strategy whether survey or population-based. This requires treating the standard persons by variables matrix in which survey data are coded and made available to analysts as an example of a two-mode network. This is a proposal that has been made before (Borgatti and Everett, 1997), but the theoretical and measurement implications of which have only recently begun to be fully taken seriously in the sociological study of cultural choice (see Boutyline, 2017; Boutyline and Vaisey, 2017; Goldberg, 2011; Lizardo, 2014; Silver et al., 2016; Vlegels and Lievens, 
2017). This paper is meant to be a contribution to this emerging line of work.

I show that rethinking the traditional arts participation or cultural choice survey data available to researchers in the sociology of taste as a relational data source can provide us with more powerful ways to tackle perennial issues in the study of cultural stratification. Especially those related to theorizing the sources and consequences of substantively relevant heterogeneity in patterns of cultural choice (Peterson, 1992), as well as the way in which persons engage cultural objects are modulated by the (perceived) relational properties of those objects (Lieberson, 2000).

A key payoff of the analysis is the demonstration of how one of the core organizing constructs of recent work in the sociology of taste-Peterson's (1992) notion of "cultural omnivorousness" - can be thought of as a special case of an entire range of conceptually related metrics useful for formally quantifying the cultural propensities of persons as marked by the properties of the genres that they consume. I show that an analogous set of metrics can be developed to differentiate the relational status of cultural genres based on the cultural choice propensities of their audiences. Iteratively applying this "reflective" strategy (Hidalgo and Hausmann, 2009), leads to the development and elucidation of a set of ordinal scales capable of extracting information encoded in the indirect paths linking linking persons and genres in the two-mode cultural network. This reveals how the ranking of persons along a given dimension of taste (popular or niche; omnivore or univore), as given by their pattern of cultural choices, mutually specifies the position of genres both in relation to each other and to the persons who choose them (Breiger, 1974).

\section{Two-mode centralities and the method of reflections}

I begin by considering the $n \times k$ matrix of persons by variables making up the usual survey data set in the sociology of taste. The same considerations apply to population-level "big" data sets not produced by sampling, as long as they are structured in a persons by variables format. Here $N$ is the number of persons (the sample size) and the $K$ is the number of of cultural items to be included in the analysis. Persons connect to cultural items by providing reports of engagement, taste, and so on. While not usually thought of in this 
manner, survey data is simply an example of a two-mode network in which persons are connected to the items they provide information on (Borgatti and Everett, 1997).

Standard matrix transformations of the data (Breiger, 1974), such as the projection of the original two-mode data set into a single-mode representation made up of an $n \times n$ weighted matrix of persons connected to other persons (in terms of the number of cultural choices they have in common) or an $k \times k$ matrix of cultural items connected to other cultural items (in terms of the number of persons shared) make the usual techniques of network analysis applicable (Borgatti and Everett, 1997; Lizardo, 2014). In the context of the usual arts participation survey, persons are connected to the cultural items they report liking or the cultural activities they report engaging in (DiMaggio, 1987).

\subsection{Person-level centrality}

Focusing on the rows of the rectangular matrix (persons included in the survey), the traditional network-analytic notion of degree centrality (Freeman, 1978) has a straightforward analogue in this context (Faust, 1997). It is the sum of the number of cultural choices the person makes:

$$
d_{i 0}=\sum_{k} a_{i k}
$$

Where $a_{i k}=1$ if person $i$ reports liking or engaging in cultural activity $k$ and the sum is over all genres $k$. This is naturally a straightforward index of cultural omnivorousness. This indicator of degree-centrality has also been referred to as "omnivorousness by volume" in the literature, and has been shown to have high levels of construct and criterion validity (Warde et al., 2008; Fishman and Lizardo, 2013).

\subsection{Genre-level centrality}

In the very same way in which persons are differentiated by the number of cultural choices they make (Peterson, 1992), genres are differentiated by the number of persons who 
choose them; this partition separates genres by their relative popularity into "niche" and "popular" genres (Lieberson, 2000; Mark, 2003).

In the standard survey data format, genre popularity is simply the proportion of the total sample who chooses that genre. In the context of two-mode network data analysis, this is the equivalent to the relative "expansiveness" or coverage of the "event" - when the matrix considered is person by event data-represented in the other mode weighted by the total number of persons (Faust, 1997):

$$
\delta_{k 0}=\frac{1}{N}\left[\sum_{i} a_{i k}\right]
$$

Where $a_{i k}=1$ if person $i$ reports liking or engaging in cultural activity $k$ and the sum is over all persons in the sample.

\subsection{Method of reflections}

The measures of person-level and genre-level centrality discussed so far $\left(d_{i 0}, \delta_{k 0}\right)$, form the basis of research and theory in the sociology of taste, in particular those reflecting the relative expansiveness of persons in the omnivorousness literature (Fishman and Lizardo, 2013; Warde et al., 2008). A key analytic advance proposed by Hidalgo and Hausmann (2009) is that, in the context of two-mode network analysis, rather than having to limit ourselves to a consideration of these "first level" metrics, they can be used as the starting point of a "reflective" measurement strategy taking advantage of the structured relational information contained in the persons by items network. In essence we can define a set of recursive (e.g. mutually definitional) indices giving us substantively meaningful information both about the structure of cultural choices (from the person's point of view) and about the structure of audience composition (from the genre's "point of view"). Some of these quantities have intuitive interpretations as basic dimensions of differentiation among both persons and genres highlighted in sociological approaches to the study of taste and cultural choice. 


\subsubsection{Average popularity of cultural choices}

Following the duality principle (Breiger, 1974; Hidalgo and Hausmann, 2009), we can use the first order classification of genres into "popular" and "niche" to generate a second-order classification of persons based on the quantity defined in equation 2 . In this way, from the person's viewpoint the most natural extension of the notion of "degree" (omnivorousness) is one using information on the relative expansiveness of cultural genres to differentiate respondents not based on the number of cultural choices they make, but based on the average popularity of the genres they choose:

$$
d_{i 1}=\frac{1}{d_{i 0}}\left[\sum_{k} a_{i k} \delta_{k 0}\right]
$$

While $d_{i 0}$ partitions the set of persons into the standard classification of "omnivores" (those who make a lot of cultural choices) versus "univores" (those who restrict themselves to a few genres), $d_{i 1}$ partitions the set of persons into "popular culture seekers" (e.g. those who tend to choose genres chosen by a large number of others) and "niche culture enthusiasts" (those who tend to choose unpopular genres). Higher values on this second order centrality thus can be taken as an index of choice bias favoring popular forms. This tendency has been referred in the literature as the "taste for popularity" (TFP) (Lieberson, 2000). ${ }^{1}$ Individuals differences and contextual factors shifting TFP upwards or downwards have been shown to have powerful effects on the dynamic evolution of the macro-level distribution of cultural choices in certain populations (Obukhova et al., 2014).

\subsubsection{Average omnivorousness of genre audiences}

Just in the same way in which we can characterize persons using information on the range of choices they make into "omnivores" and "univores" (equation 1), or into those displaying a choice bias towards either popular or niche forms (equation 3) we can use the

\footnotetext{
${ }^{1}$ Note that the obverse quantity (a "taste for unpopularity") also has a substantive interpretation as an attempt to seek rare cultural forms uncontaminated by the mass (Arsel and Thompson, 2011).
} 
duality principle and loop back and characterize genres by the relative omnivorousness of the persons who choose them:

$$
\delta_{k 1}=\frac{1}{N_{k}}\left[\sum_{i} a_{i k} d_{i 0}\right]
$$

Where $N_{k}=\sum_{i} a_{i k}$. While $\delta_{k 0}$ partitions genres into "common" and "niche" genres (Warde et al., 2008), $\delta_{k 1}$ partitions genres into "those preferred by omnivores" and "those preferred by univores." It is easy to verify intuitively these are two empirically distinct classifications, since it is possible that a given genre may have the comparable levels of popularity as another genre, yet actually have an audience more heavily composed of omnivores than another genre (as we will see below).

\subsubsection{Average audience omnivorousness of cultural choices}

Following the same approach we used to characterize the cultural choices made by persons in terms of the relative popularity of the genres chosen, we can use the classification of genres obtained by using equation 3 to recursively classify persons by the relative audience composition - in terms of omnivores and univores - of the cultural choices they make:

$$
d_{i 2}=\frac{1}{d_{i 0}}\left[\sum_{k} a_{i k} \delta_{k 1}\right]
$$

This time rather than partitioning the set of persons into omnivores and univores (as is done by $d_{i 0}$ ), or popular and niche culture enthusiasts (as is done by $d_{i 1}$ ), the quantity computed in equation 5 partitions persons into those who prefer the cultural genres that are heavily chosen by omnivores versus those who gravitate towards those genres that are more likely to be chosen by univores.

\subsubsection{Average popular choice bias of genre audiences}

The reader may have already anticipated that we can take this one step further and classify genres according to this last classification of persons: 
Table 1: Substantive interpretations of key quantities of interest produced by the method of reflections (genre-level quantities in gray-shaded rows)

\begin{tabular}{|l|l|l|l|}
\hline \hline$d_{i 0}$ & Working Name & Short Summary & Question Form \\
\hline$\delta_{k 0}$ & Popularity & $\begin{array}{l}\text { Pumber of genres chosen by } \\
\text { person } i\end{array}$ & $\begin{array}{l}\text { How many genres are chosen } \\
\text { by person } i ?\end{array}$ \\
\hline$d_{i 1}$ & $\begin{array}{l}\text { Popular Choice } \\
\text { choose genre } k\end{array}$ & $\begin{array}{l}\text { Average popularity of the } \\
\text { genres chosen by person } i\end{array}$ & $\begin{array}{l}\text { How popular are the genres } \\
\text { chosen by person } i ?\end{array}$ \\
\hline$\delta_{k 1}$ & Genre Omnivo- & $\begin{array}{l}\text { Average omnivorousness of } \\
\text { the fans of genre } k\end{array}$ & $\begin{array}{l}\text { How omnivorous are the } \\
\text { persons who choose genre } k ?\end{array}$ \\
\hline$d_{i 2}$ & $\begin{array}{l}\text { Omnivore Choice } \\
\text { Bias }\end{array}$ & $\begin{array}{l}\text { Average audience omnivo- } \\
\text { rousness of the genres cho- } \\
\text { sen by person } i\end{array}$ & $\begin{array}{l}\text { Does person } i \text { prefer genres } \\
\text { chosen by omnivores? }\end{array}$ \\
\hline$\delta_{k 2}$ & $\begin{array}{l}2^{\text {nd }} \text { Order Popu- } \\
\text { larity }\end{array}$ & $\begin{array}{l}\text { Average popularity of the } \\
\text { genres chosen by persons } \\
\text { who choose genre } i\end{array}$ & $\begin{array}{l}\text { Are persons who choose } \\
\text { genre } k \text { likely to choose pop- } \\
\text { ular genres? }\end{array}$ \\
\hline
\end{tabular}

$$
\delta_{k 2}=\frac{1}{N_{k}}\left[\sum_{i} a_{i k} d_{i 1}\right]
$$

Which differentiate genres not according to relative popularity, or according to the relative omnivorousness of their audiences, bu this time by the relative likelihood that their audiences are composed of persons who choose genres which are common or niche. That is, this gives us a sense of the two-step popularity of a given genre; even if not chosen by a large number of people, cultural forms may be indirectly popular if the people who choose them also tend to choose other popular forms. In the same way, a popular genre may have second-order niche status if the persons who choose them also tend to choose niche genres. 


\subsubsection{Higher order reflections}

Naturally, we can keep iterating this process $M$ times to obtain further quantities of interest at both the person $\left(d_{i 3}, d_{i 4}, d_{i 5} \ldots d_{i M}\right)$ and genre $\left(\delta_{k 3}, \delta_{k 4}, \delta_{k 5} \ldots \delta_{M}\right)$ levels. For instance, high versus low values of $d_{i 3}$, classifies persons into those who prefer genres whose audiences are composed primarily of persons who choose popular genres versus those who gravitate towards genres whose audiences are composed of niche culture enthusiasts (as given by equation 6 ). The quantity $\delta_{k 3}$ on the other hand, would give us a classification of genres separating those whose audiences are primarily composed of people who, while not necessarily themselves omnivorous, gravitate towards genres chosen by omnivores versus those whose audiences are composed of people who prefer genres which are preferred by univores (as given by equation 5 ).

In principle, as $M \rightarrow \infty$, further reflections begin to converge around the same value and the centrality ranks of people and genres across each reflection begin to freeze. In practice, convergence occurs after a relatively small number of iterations depending on network size (e.g. $M \approx 16$ ) (Hidalgo and Hausmann, 2009). This is the case for these data. The correlation between the standardized centrality ranks at the 14th and 16th reflections and at the 13 th and 15 th reflections at the level of both people and genres is $\approx 0.99$, suggesting that the centrality ranks are "frozen" by this point and that further reflections do not add novel information.

Table 1 provides a summary of the key lower order quantities of interest, along with their (heuristic) labels, their operational definition, and the key substantive question that they are designed to answer. These will be the focus of the analysis that follows.

\subsection{Interpreting higher order reflections}

Higher order reflections become increasingly difficult to interpret intuitively although they continue to capture information embedded in more abstract recursions across people and genres in the two-mode cultural network (e.g. "preference for genres which are preferred by those who prefer..."). One way to visualize what is captured in the higher order reflections is to imagine a "walker" who starts with a randomly chosen person and then selects a 
randomly chosen genre from that person's cultural repertoire. This takes the random walker to a particular cultural genre, upon which she selects a random fan of that genre leading her back to a person in the other mode (which may be the person with which she started!). The walker then picks a random genre from that fan's cultural repertoire, which leads her back to a cultural object in the other mode. The walker then repeats the same process $M$ times bouncing back and forth between persons and genres. If the cultural network has meaningful structure, then we can surmise that even when starting with a random person, and undergoing $M$ hops (where $M$ is a relatively big number), the walker will likely return to a person who has some sort of direct or indirect commonality (in terms of their pattern of cultural choice) with the person with whom she started (and vice versa for cultural genres).

In this respect, even small rank deviations within higher-order reflections, provide us with substantively meaningful quantities that take advantage of the systematic (e.g. nonrandom) relational structure implied in the bipartite matrix of persons by cultural items. ${ }^{2}$ Thus, higher order reflections define "communities" of people based on the (popularity weighted) similarities of the genres they choose and "communities" of genres based on the (omnivorousness weighted) similarities between the people that choose them. In this respect, the method of reflections can be thought of as one of a family of methods that use a two-mode matrix to generalize the idea of similarity between a set of objects by leveraging information from their relations to those in another set (Kovács, 2010). ${ }^{3}$

\subsection{Method of reflections in relation to other partitioning approaches}

In fact, as Mealy et al. (2018) have very recently noted, the method of reflections belongs in the family of spectral clustering algorithms for partitioning similarity matrices, and can therefore be thought of as a complement to recently proposed methods to study the structure of cultural choices that exploit relational information embedded in survey responses. These

\footnotetext{
${ }^{2}$ I would like to thank Jacob Foster for suggesting this line of interpretation and encouraging me to articulate it more clearly.

${ }^{3}$ I would like to thank John Levi Martin (email communication) for pointing to the family resemblance between the method of reflections and the Kovács approach.
} 
include relational class analysis (Goldberg, 2011), correlational class analysis (Boutyline, 2017), and belief-network analysis (Boutyline and Vaisey, 2017) among others.

As noted before, while the spirit of the reflection approach given its strong grounding in the idea of duality (Breiger, 1974), is closer to that of Kovács (2010), the result is a multiple trajectory of linear orderings that can be used to define clusters of persons and genres. As such, it incorporates the analytic goals of the "class analysis" types of methods. However, rather than using substantive features of the pattern of choices between people (e.g. the correlation distance or relationality between response patterns at the person-level), similarity between people emerges purely as a result of the average topological similarities between the genres that people choose (e.g. average genre popularity, average first order omnivorousness, and so on). The connection between the resulting rank of people (or genres) and substantive cultural choices (or genre demographic composition) can then be made into an empirical question as we will see below.

\subsection{Reflective and eigenvector centralities}

Finally, it is important to clarify the relationship between the method of reflections and other ways of defining rank centralities between two sets of objects based on them being connected (directly or indirectly) to central objects in another set. The core contribution here is Bonacich's (1991) extension of the notion of eigenvector centrality to the two mode case. As Bonacich showed, the leading eigenvector obtained from the singular value decomposition of the affiliation matrix will produce such simultaneous centralities for both persons and cultural forms (in the present case) such that central people will be those who choose central (e.g. popular) cultural forms and central cultural forms will be those that are chosen by central (e.g. omnivorous) people (Faust, 1997).

As such, a fair question is whether the reflective approach is just a cumbersome way to obtain such a ranking given the conceptual similarity between the eigenvector and reflective centrality notion. In fact, this is an issue that has already come up in the literature, with some arguing that a reflective strategy is an algorithm for calculating a version of eigenvector centrality (Morrison et al., 2017). However, as Mealy et al. (2018) note in a recent 
contribution. This interpretation, while intuitive, is not on the right track. The key issue is that the eigenvector solution takes as its input the unweighted affiliation matrix, while the reflective strategy weights each iteration by the centrality of each mode (in taking an average of averages). As a result, the eigenvector rank will be strongly dependent on the first order centrality of both people (omnivorousness) and genres (popularity), while the reflective equilibrium rank can be shown to be orthogonal to, although typically correlated with, the first order centralities of each mode. In this respect, the eigenvector centrality score does not add much information beyond first order omnivorousness and popularity with the correlation between these two quantities being to 1.0, while the correlation between eigenvector and the equilibrium reflective centrality rank between much lower (see Mealy et al., 2018, , Figure 2). ${ }^{4}$ As such, reflective centrality is picking up something different from the eigenvector idea both conceptually and empirically.

\section{Empirical Analysis}

In the following analysis, I demonstrate the substantive relevance of the method of reflections for analyzing the type survey-based cultural choice data typical of studies in the sociology of taste. The goal is to demonstrate that applying duality-based network techniques to this sort of data allows us to recover insights that would be overlooked using traditional (e.g. general linear model-based) approaches.

The data come from a survey on the cultural habits and tastes of Americans fielded in the summer of 2012 (see Lizardo and Skiles, 2015, 2016a,b). The data were collected by Survey Sample International (SSI), a private firm that specializes in sampling, data collection, and analysis. SSI managed recruitment and participation invitation tasks to generate a panel of adults from which our cross-sectional sample was drawn. Survey respondents were selected from the panel for participation on the basis of age, gender, race, education, and geographic

\footnotetext{
${ }^{4}$ This is a result that is reproduced in these data. The correlation between the genre eigenvector centrality computed on the original two mode matrix and $\delta_{k 2}$ is 0.96 , the correlation between eigenvector centrality and $\delta_{k 16}$ attenuates to 0.48 .
} 
region to approximate a sample representative of the U.S. population $(\mathrm{n}=2,250)$. The survey asked respondents to report whether the regularly listened to music classified into 20 categories of musical styles: classical or symphony and chamber, opera or operetta, jazz, Broadway musicals or showtunes, mood or easy listening, big band or swing, classic rock or oldies, country, bluegrass, folk, hymns or gospel, Latin, Spanish or salsa, rap or hip hop, blues or R\&B, reggae, top 40 or pop, contemporary rock, indie or alternative rock, dance, club or electronic, and hard rock or heavy metal.

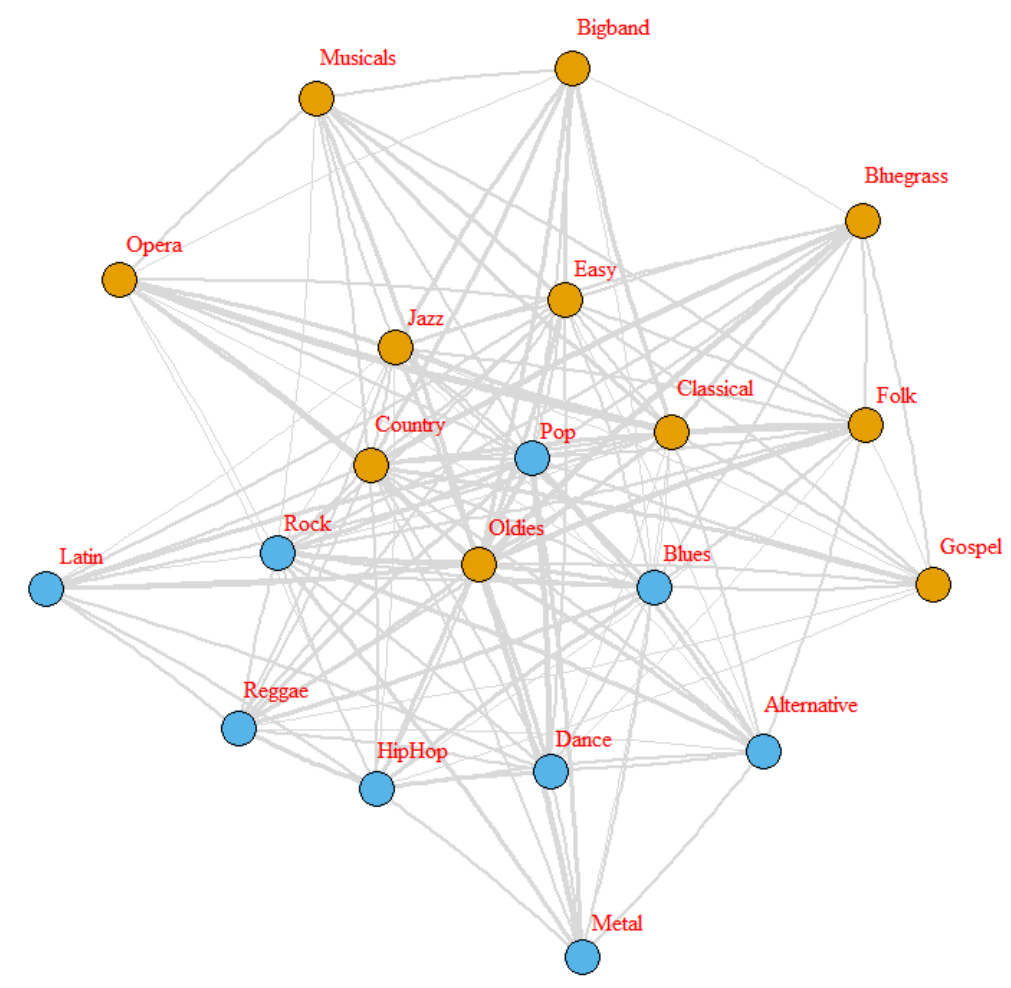

Figure 1: One-mode projection of musical genres based on their audience overlap. For each pair of genres $(j, k)$ the overlap is given by taking the audience of genre $j$ and dividing it by the minimum of the two genre audiences. To improve readability, only overlaps above the 25 th percentile are considered. Edge-width is proportional to the level of audience overlap. Nodes are colored by their community membership obtained from a leading eigenvector partitioning of the modularity matrix (Newman, 2006). 
After deleting respondents who did not answer all of the cultural preference questions and respondents who did not report listening to any of the genres (these are "isolates" in the cultural network), we are left with a rectangular matrix is of dimensions $2,042 \times 20$, with the number of rows indexing the number of respondents included in the sample. This matrix is coded so that it contains only binary entries with $a_{i k}=1$ if the $i^{\text {th }}$ respondent reports regularly listening to the $k^{t h}$ musical genre. This is the matrix representation of the two-mode cultural network linking persons and genres.

A one-mode projection - using the standard approach developed by Breiger (1974) — of the columns of this matrix (representing the genres) is shown in Figure 1. In the figure, genres are connected based on their relative levels of audience overlap. The figure reveals an intuitive arrangement with a binary community structure separating "young" industry-based genres (e.g. Pop/Top-40, Hip Hop, Metal, etc.) from more traditionally legitimated high status and folk offerings. Towards the middle of the graph (and thus accounting for the bulk of the connectivity in the network), we see the most "popular" (in terms of overall audience size) genres (e.g. Country, Oldies/Classic Rock, Pop, etc.). These are genres that display the most variegated patterns audience overlap with other genres. Towards the periphery we see "niche" genres that only overlap with a select subset of other genres, such as Opera, Metal, Gospel, Bluegrass, and Big Band.

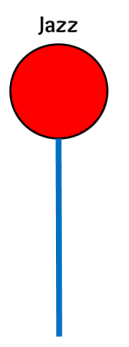

Harry

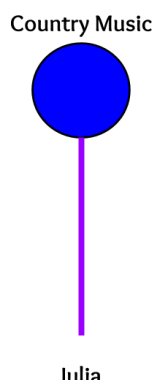

(a) Data before permutation

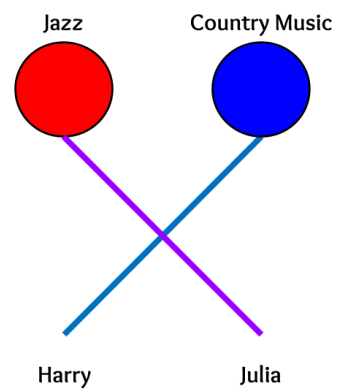

(b) Data after permutation

Figure 2: Randomization algorithm used to generate null comparison data (Maslov et al., 2004). 


\subsection{Constructing a baseline for comparison}

In what follows, it is important to ascertain which features of the cultural-network are due to intrinsic biases governing the way that persons select cultural genres, and which ones result merely by chance given the original distribution of both the number of choices at the personal level and the distribution of popularity across genres.

To do this I construct a randomized data ensemble set preserving the distributional properties of the original data, but removing all remaining structure. I rely on a simple link-permutation procedure designed to preserve both the distribution of popularity across cultural forms and the distribution of omnivorousness across persons but make everything else random (Maslov et al., 2004). This is accomplished by cycling through the network, selecting two persons at random (Harry and Julia) and for each person selecting an existing edge connecting that person to a musical genre and the swapping the two edges.

For instance, in Figure 2a, Harry listens to jazz and Julia listens to country music. Provided that neither the [Julia $\leftrightarrow$ jazz] nor the [Harry $\leftrightarrow$ country music] links exist, we simply swap the original links, deleting Julia's choice of country music and giving her a taste for jazz while deleting Harry's original choice of jazz and giving him a taste for country. It is easy to verify that this procedure preserves the original number of choices across persons and the original popularity of each cultural genre (the omnivorousness of Harry and Julia and the original popularity of jazz and country are preserved, but randomizes the choice process.

To construct the baseline data set used below, I begin with the original data and repeat this procedure for 100,000 trials. Each of these runs produces a "data set" with randomized links. I create an ensemble of 500 such data sets and take the average of each of the observed quantities of interest $\left(d_{i}\right.$ 's and $\delta_{k}$ 's) to serve as a baseline for comparison against the same quantities computed in the original data. 


\section{Results}

\subsection{Genre-level analysis}

\subsection{1. $\delta_{k 1}-\delta_{k 0}$ plot}

I begin by using the method of reflections to gain insight into the structure produced among genres as a result of person-level patterns of cultural choice. A key analytic tool here is the $\delta_{k 1}-\delta_{k 0}$ plot (Hidalgo and Hausmann, 2009). This plots genre popularity against the relative audience composition of the genre based on person-level first-order centrality in the cultural network (average omnivorousness of those who choose the genre). Figure 3a shows the $\delta_{k 1}-\delta_{k 0}$ plot for the original data. The regression line running across the plot represents the linear relationship between $\delta_{k 0}$ and $\delta_{k 1}$ in the randomized version of the data, and thus serves as a point of reference.

Note that the distribution of genres in the $\delta_{k 0}-\delta_{k 1}$ space suggests that there is a strong negative trade-off between being a popular genre and being a genre whose audiences are composed by omnivores $(r=-0.83)$. Note that this correlation, while steep, is actually smaller than we would expect in the absence of structured biases in the ways that persons select certain genres; in fact the results from the null-model suggests that these two quantities should not be as distinct as they are in the original data set, but should be instead perfect functions of one another $(r \approx-1.0)$.

This means that deviations from this pattern are informative. Note for instance, that while Dance Music and Gospel have similar levels of popularity $\left(\delta_{k 0}\right)$, fans of dance music are more likely to be omnivores $\left(\delta_{k 1}\right)$ than fans of gospel. The same contrast (at different levels of genre omnivorousness and popularity) applies to Pop and Country, Jazz (or Blues) and Hip Hop, Alternative Rock and Latin Music, and Folk and Opera fans (respectively). The space divides genres into different groups both diagonally (those located in the upper left quadrant versus those located in the lower right quadrant) and vertically (those above the null regression line and those below it).

The first division separates genres with relatively small audiences whose fans tend to select a large number of genres from those with relatively large audiences whose fans are 
more univorous. Foremost among the former group are folk music, bluegrass and opera, while genres representative of the latter group are pop music, country music, and classic rock. The vertical distinction separates genres who have more audience omnivorousness than expected given their popularity (disproportionately attracting omnivores), from those that have less (disproportionately attracting univores). On the negative side, we find such cultural forms as gospel or religious music, hip hop, Latin music, and classical music. On the positive side - genres whose audiences contain many more omnivores than expected give their audience size - we find such cultural forms as folk music, bluegrass, dance, jazz, and blues.

\subsubsection{Relationships between first order and higher order reflective centralities}

As we saw above, the method of reflections yields a series of dimensions of genre popularity and genre omnivorousness beyond the quantities observed at the first and second step of the reflection process. Accordingly, second order genre popularity is the average of the average popularity of the cultural choices of its audience: that is the "indirect" popularity that a genre gets by virtue of being two steps away from other genres whose audiences are attracted to popular choices, and so on for higher order levels of popularity. These are the even values of $\delta_{k m}$ for all $m=2,4,6, \ldots M$. The same goes for genre omnivorousness: a genre may not have a lot of omnivore fans, but it is possible that the people who choose that genre are likely to choose genres that are chosen by omnivores. These are the odd values of $\delta_{k m}$ up to $M$.

As noted earlier, higher order reflections of genre popularity and genre omnivorousness scores should reflect the result of "longer" random walks going back and forth from persons to genres along the structure provided by the connectivity of the two-mode cultural network (Hidalgo and Hausmann, 2009). In contrast to the randomized network, the structure of the real network should be systematically shaped by whatever cultural and social logics "bias" the cultural choices of persons and the groupings of genre audiences (DiMaggio, 1987). Accordingly, we should expect that the correlation between first and higher order quantities to attenuate as a function of the number of steps traversed $(m)$ in the original network, 
but not in the randomized network. The differences between these correlations thus carry information about implicit systematic structure encoded in the two-mode cultural network.

This is precisely what is demonstrated in the correlations plots shown Figures 4a and 4b. While the correlation between first $\left(\delta_{k 0}\right)$ and second order $\left(\delta_{k 2}\right)$ genre popularity is $r=0.97$, that estimate declines to $r=0.54$ for the $12^{\text {th }}$ order realization of the same quantity. If people made random genre choices we would expect this last correlation to be on the order of $r \approx 1.0$. The same goes for corresponding link between first and higher-order realizations of genre omnivorousness. In the original data, the link between first $\left(\delta_{k 1}\right)$ and second $\left(\delta_{k 3}\right)$ order genre omnivorousness is also 0.97. But this quantity declines to 0.38 by the $13^{\text {th }}$ iteration, when it would have been closer to 1.0 if the data lacked systematic structure. This indicates that while popular genres are more likely to be connected to other popular genres via the (biased toward popularity) choices that their fans make than less popular genres, this correlation - while substantial - is much weaker than we would observe if persons made unstructured cultural choices. In fact, if this was the case, then we should be able to predict that rank order of each genre at the $m^{\text {th }}$ step of the reflection from its original popularity rank order with high-levels of accuracy, as shown in Figure 4b. The fact that we cannot do that reveals substantively meaningful biases in the ways in which person-level choices determine the audience composition of each genre.

\subsection{Slopegraphs of reflective centralities}

\subsubsection{Genre popularity reflective centralities}

As Figure 5a shows, the trajectory of genre ranks across the various reflections reveal important structure that is absent in the reshuffled data. Looking at the rank order in the initial (popularity-based ranking) and the final $\left(16^{\text {th }}\right)$ reflection, shows a substantial reorganization of the ranking of genres, suggesting that certain genres might not begin as very popular, but receive second-order popularity from their indirect connection to other popular genres via the persons who choose them. If we compare this to the patterns observed in the randomized data (Figure 5b), we can see that, we should have expected to observe no such reorganization if there was no structure in the data above an beyond the original 
distributions of personal choices and genre popularity: Most genres retain their relative rank towards the top or bottom of the distribution across the reflection space.

In the observed data, two ideal type of cultural genres emerge: those that experience an upward popularity (or audience attraction to popular forms at higher reflections) trajectory through the reflection space, and those that experience a downward decline in relative rank in the same metric. Heavy metal experiences the most dramatic improvement in rank going from the bottom to the top rank at the last iteration; this is in spite of the fact that the reshuffled data plot suggests that this genre should have experienced little or no mobility in the reflective centrality space. This type of upward trajectory is shared by alternative rock and to a lesser extent folk and bluegrass music. This last case is particularly instructive, since as shown in the bottom panel, the expected trajectory of this genre should have been the exact opposite: a steady drop to the bottom. In all, positive trajectories are indicative of genres that are not popular (or have audiences that prefer popular genres) but which are more likely to be connected to other popular genres via the choices that members of their audience make. This is indicative of an affinity of these genres to combine with (literally) "popular" culture to a greater extent than other genres in the cultural choices of persons, in spite of heterogeneity in the baseline popularity of some of these genres.

On the other hand, there are a subset of genres experiencing a steeper downward trajectory than expected by chance. This is indicative of a greater likelihood to be combined, via the direct and indirect choices of their audiences, with "niche" (small audience) genres, thus resulting in a drop in audience popular choice bias as we move up in the number of iterations. Most clearly representative of this pattern are classical, jazz, and opera (and to a lesser extent classic rock). These are the genres that are traditionally thought of as comprising the classical canon of high status (institutionally legitimated as "art") genres (Warde et al., 2008). This suggest that this type of culture tends to find affinity with less popular genres (and less affinity with popular genres) in the choices of their audiences. 


\subsubsection{Genre omnivorousness reflective centralities}

The genre rank in term of the omnivorousness metric also undergoes a fairly dramatic reorganization in the original data (Figure 6a) one that is much more radical than would be observed if nothing was going on other than the distribution of choices and popularity (Figure 6b). Just like before, we can distinguish between two ideal-typical trajectories: "upwardly moving" genres begin with low to middling levels of audience omnivorousness, but improve their ranking as we increase the iterations in the reflective centrality space. The prototypical genre in this regard seems to be hip hop, whose audience members are not very likely to be omnivorous, but which ends up towards the top of the list in the final $\left(16^{\text {th }}\right)$ iteration. This in spite of the fact that the expected (null) trajectory of this genre should be flat, as shown in Figure 6b. Latin/Spanish music follows an attenuated version of the hip hop trajectory, which is significant since the null model would have indicated declining $n^{\text {th }}$ order audience omnivorousness with increasing iterations.

This kind of trajectory seems to be indicative of an affinity to combine with genres that are favored by omnivores. In the case of hip hop, this is clear in the steep positive slope of the rank transition from $\delta_{k 1}$ to $\delta_{k 3}$ in Figure 5a: while hip hop audiences are themselves not particularly omnivorous, people who combine it with other genres tend to choose genres that are favored by omnivores. This is consistent with the idea that, in some cases, when omnivores add additional genres to their repertoire they (selectively) choose genres whose audiences may themselves be composed of univores (and which thus form "crisp" cultural categories) as a way to expand their cultural repertoire (Goldberg et al., 2016).

A second ideal typical trajectory through the genre-omnivorousness rank space is one of "downward mobility." These are genres whose base audience is composed of people who also select a relatively large number of genres, but which are indirectly connected to genres whose audience tend to be univores. Most clearly exemplifying this pattern are folk music and bluegrass, who begin in the top rank of genre omnivorousness, but which end up towards the bottom by the last iteration. The pattern for folk music is particularly striking given that, as shown in Figure 6b, the baseline expectation (holding the original distributions of omnivorousness and popularity constant) should have been flat. Other 
genres that experience downward mobility in the omnivorousness reflective ranks when we should have expected none are big band, opera, and musicals. This pattern is indicative of an elective affinity between these cultural forms and genres preferred by univores, a pattern realized via the direct and indirect choices of their audience members.

\subsubsection{Criterion validity of higher order genre ranks}

The reader may wonder whether the ultimate genre rankings at the later steps of the reflection process carry substantive meaning. That is, do we obtain novel information about each cultural genre by ranking them according to higher order $\delta_{k}$ 's that would not be available using lower order values? This question is valid given the fact that we pay a price (in terms of intuitive interpretation) when considering higher order values.

Figure 7 shows that the higher order ranking reveal information about each genre that would not be predictable from lower order realizations. For instance, Figure 7a shows that the correlation between the educational composition of a genre, as given by the proportion of its audience that reports having finished their education at the high school level, and its overall popularity is not very strong $(r=0.20)$. This is inconsistent with the idea that people who with relatively lower levels of cultural capital are attracted to the most "common" forms of culture in the United States (Holt, 1998).

However, as shown in Figure $7 \mathrm{~b}$, the correlation between the educational composition of a given genre and its audience popularity bias at the $15^{\text {th }}$ step is very strong $r=0.78$. Genres with majority non-college educated audiences also happen to be the ones indirectly connected to more common forms via the choices of its fans. In effect, the reorganization of the genre ranks at this step reveals demographic differentiation at the genre level that is occluded in the raw popularity measure, but that is embedded (and thus recoverable) in higher-order reflections.

Figures $7 \mathrm{c}$ and $7 \mathrm{~d}$ show a similar dynamic for the connection between lower and higher order genre omnivorousness and the racial diversity of the genre's audience. ${ }^{5}$ As shown

\footnotetext{
${ }^{5}$ At the genre level, racial diversity is measured using Blau's index of heterogeneity $\left(1-\sum_{i} p_{i}^{2}\right)$ computed using the proportion White, Black, Hispanic, and Asian respondents who report listening to music in that
} 
in Figure 7c, there is no correlation between how racial diverse a genre's audience is and the average omnivorousness of that genre's audience. This would seem to go counter the supposition that there is an aggregate connection between omnivorousness and a taste for multicultural diversity at the level of cultural forms (Bryson, 1996; Ollivier, 2008).

However, as shown in Figure 7d, the correlation between the ethnoracial diversity of genre audiences and the higher order omnivorousness bias of a genre is strong and in the expected direction $\left(r=0.64\right.$; attenuated by the outlying status of Gospel/Religious music: $r^{- \text {gospel }}=$ 0.77). The more racially diverse a genre's audience, the more likely it is that a genre's audience members the larger the indirect omnivore choice bias of that genre's audience. Once again, this result reveals that higher order rankings of genres across the reflective centrality landscape reveals sociologically important features of genre differentiation that would remain hidden in lower order realizations.

\subsection{Individual Level Analysis}

As noted earlier, we can obtain reflective centralities quantities at the individual level that are analogues of those that we have considered at the genre level. One complication when looking at this information across persons is the number of cases to be considered. While in the preceding we had $N=20$ genres, at the level of individuals we have $N=2,042$ cases. This yields as many individual trajectories across the reflective centrality space, both in Popular Choice Bias (odd numbered centralities) and Omnivore Choice Bias (even number centralities), rendering the direct plotting strategy used for genre analysis less useful.

\subsubsection{Growth Mixture Modeling of Individual Centrality Trajectories}

To simplify the analytic task, I treat information on the trajectories of persons across the reflective centrality space, as equivalent to temporal trajectories in growth curve models. This multilevel or "mixed" general linear modeling strategy is useful when information is available on some individual outcome at multiple points in time (thus forming a "trajectory") such as grades, income, educational status and so on. The task is then to come

genre. 
up with "clusters" of individuals who share statistically indistinguishable "trajectories." In this application, rather than having trajectories in time, we have trajectories in reflective centrality space. To that end, I subject the data set formed by individuals and their centrality trajectories to a form of non-parametric latent growth analysis called growth mixture modeling (Jung and Wickrama, 2008).

The idea behind growth mixture modeling is that individuals are thought to belong to a finite number of latent-classes that generate their specified trajectory through the centrality space. For both Popular and Omnivore Choice bias, I fit a growth mixture model specifying four latent classes of persons, with each of the four time trajectories fitted parametrically using a quartic polynomial (to allow for arbitrary movement within the space). We then assign individuals to each latent class, and examine the correlates of latent-trajectory assignment using a multinomial logit regression with socio-demographic variables as the main predictors. To keep matters brief, I focus on two of the most important individual level predictors in the sociological literature on taste: age and education.

The results of the analysis is shown in Figure 8. Figure 8a shows a spaghetti plot of all individuals trajectories through the omnivore choice bias space colored by their latentclass assignment category. The first group (plotted in red) is composed of a relatively small number of individuals (about $11 \%$ of the sample) who make a small number of initial genre choices (thus qualifying as "univores"). These choices also happen to be indirectly connected to genres that are avoided by omnivores as revealed by the declining omnivore choice bias across further reflections. The second group, making up about a third of the sample (plotted in green), makes a middling number of initial choices but choose genres that are not chosen by omnivores. This cluster can be contrasted with a relatively a smaller group (about $15 \%$ of individuals), plotted in purple, whom, while not making a large number of choices initially (just as the second group), can be shown to display very sharp levels of (indirect) omnivore choice bias as we move toward higher order reflections. These are the individuals who seem to be most attracted to genres chosen by omnivores or indirectly connected to such genres. Finally, we have a fourth large (42\%) group of individuals, plotted in teal, some of whom who seem to display high level of first order omnivorousness but which choose 
genres that are not necessarily chosen by omnivores (as shown by the flat to downward trend across the centrality space); as we will see below this pattern seems to correspond to the more established (young and middle class) pattern of omnivorousness as a form of cultural capital.

In the case of popular choice bias (Figure 8b), we observe somewhat similar, but not quite identical results. First, we find a relatively small group (14\%), plotted in red, made up of individuals who make relatively unpopular genre choices and whose popular choice bias actually declines as we consider higher order popular choice bias reflections. This is followed a much larger group (38\%), plotted in green, of persons who make choices of middling popularity and which stay at that level through the higher order reflections. We then have a similar-size group (34\%) of persons who make choices similar to those of the last group initially, but whose indirect popularity choice bias increases as we consider higher order reflections. Finally, we have a group of similar size as the first (14\%) who select the most popular genres initially, but whose popular choice bias declines as we move up the reflective centrality space.

\subsubsection{Criterion validity of centrality trajectory groupings}

The reader may agree that we can induce statistical groups based on the trajectory of individuals across the omnivore and popular choice bias reflective centrality spaces but may be skeptical as to substantive relevance of this clustering exercise. To ascertain whether the "groups" induced from the growth mixture model are substantively meaningful, I computed the marginal probabilities obtained from a multinomial logit model predicting latent growth curve class assignment from individual sociodemographic characteristics. In the model respondents age and education are allowed to have non-linear effects by including linear and quadratic versions of the ordinal age and education input variables as predictors while varying within levels of one another (by including multiplicative combinations of the linear and quadratic age and education inputs as additional predictors). Models adjust for respondent's gender and racial identification category by including additive binary indicators of gender (male and female) and race categories (white, black, asian, hispanic, and other) as 
additional class-assignment predictors.

Figure 9a plots the results of the class assignment analysis based on the omnivore-choice bias centrality trajectory. The results show that the four latent groups encode substantively meaningful information on both class rank distinction (pitting the higher educated versus the lower educated and vice versa) and generational processes (pitting the young versus the old and vice versa). The bar charts shown as Figure 10 plot the responses to an item asking respondents to list their favorite musical genre from the same list of twenty that we have considered. These analyses demonstrate that clustering individuals by their trajectory through centrality space recovers not only coherent sociodemographic "classes" but also decipherable "taste cultures" in the traditional sense (Gans, 1999).

Recall that the first group in the omnivore choice bias trajectory plot (Figure 8a) was a relatively small set of univores who remained indirectly disconnected from genres chosen by omnivores. As the first panel of Figure 9a shows, the best predictor of being in this group is having low levels of education, and being older. Thus, univores who select genres avoided by omnivores tend to belong to an older segment of the working class. The first panel of Figure 10a sheds light on the substantive tastes of this group: these are persons that gravitate primarily as to Country, Classic Rock/Oldies and Religious Music or Gospel as their preferred genres, thus demonstrating a fairly traditional approach to aesthetics characteristic of persons with low endowments of cultural capital (Holt, 1998). Both the demographic and taste-based correlates are thus consistent with previous work on "univorousness" as a social form (Bryson, 1997; Holt, 1998).

Persons in the first category stand in contrast to the those depicted in the third panel; recall that this is the group that began with very high levels of first order omnivorousness, but who connects to genres less likely to be preferred by omnivores (indirectly) via higher order reflections. The analysis reveals that, consistent with previous work, the best predictor of this pattern is middle class status (as given by levels of education) and being young. These group thus exhibits the precise mirror image (in terms of class rank and age-opposition), one that is consistent with the notion of "omnivorousness" (in this case, "indirect omnivorousness") as an established form of cultural capital characteristic of younger segments of the 
upper-middle class (Lizardo, 2008). Consistent with this assessment, this group displays the most variegated distribution of favorites, with no one genre dominating over any other, and with many genres that are preferred by small minority of respondents receiving relatively large proportion of nominations (third panel of Figure 10a). The key logic of taste for this group is to begin with genres that have a high probability of being consumed by omnivores and then move to genres that are more likely to be preferred by univores to round out their diverse repertoire.

The second and fourth panels of Figure 9a, on the other hand, reveal (mirror image) age effects without much class opposition. As shown in the second panel, the best predictor of being assigned to the group that starts with middling levels of omnivorousness and stays that way through higher reflections is being old regardless of educational status; this group contrasts with those respondents shown in the fourth panel for which the best predictor of class assignment is being young.

This last set of persons is the group (see Figure 8a) who is likely to select genres that are also selected by omnivores even when making a relatively small number of initial choices. The Fourth panel of 10a shows that this last group of respondents tends to gravitate towards a host of contemporary genres, such as Rap and Hip Hop, Alternative Rock, R\&B, and Latin. In this respect, they can be said to possess a form of emerging "multicultural capital" (Bryson, 1996; Ollivier, 2008). Their choices contrast with their older counterparts in the second group, whose choices seem to be an attenuated version of the traditional omnivorousness of the first group of respondents.

Looking at the predictor of latent class assignment from the popularity bias trajectories, we find a formally analogous pattern of results, with two groups exhibiting a relatively class neutral age-related opposition, and other two groups reflecting a marked class rank that is partially independent of age. The key difference is that the two groups showing a clear difference in the predictive power of education also demonstrate a non-monotonic pattern of age-differentiation, pitting both extreme ends of the age distribution against those of middle age.

Looking at the first and third panels of Figure 9b, reveals that the first and third latent 
growth clusters reveal oppositions primarily structured by age and secondarily by education. Thus, we find that the group that selects the least popular genres from the get go (as given by $\left.d_{i 1}\right)$, with those genres also being indirectly connected to less popular genres in higher order reflections, tend to be older and of high educational status. This group stands in contrast to younger, less educated people (third panel) who exhibit high popular choice bias early on, which only becomes accentuated in higher order iterations.

The taste choices of these two groups correspond to the intuition derived from their sociodemographic composition. As shown, in Figure 10b, the older, more highly educated group is disproportionately more likely to be attracted to traditionally legitimated genres, thus representing a form of "highbrow" taste pattern. These include such forms as classical and to a lesser extent Jazz and Opera. The third latent growth group is by contrast characterized by a heightened chance to choose most "rock" genres as their favorite (Classic, Contemporary, Alternative, or Pop) which is consistent with their bias toward popular genres in their initial choices and the augmentation of this bias in higher order reflections.

Looking at the second and fourth panels of Figure 9b, we can see that the best predictor of being in the latent growth group that is more likely to select genres that are not very popular (but which are also not the least popular) and which stays that way through higher order reflections, is education, with either younger or older college-educated people being more likely to fall into this category. Looking at the taste choices of this last group in Figure 10b, reveals an "eclectic" distribution of favorites (no genre dominant) indicating that "omnivores" may be most likely to be found in this group. Essentially, their popular choice bias is in the middle range precisely because omnivores combine popular and non-popular genres, which works out to a "middling" popular choice bias (on average). This group can be contrasted to the relatively small cluster of people who pick the most popular genres from the get-go. The analysis reveals that this last set of persons is be composed mainly of low education, middle-aged people who are disproportionately attracted to the most popular (by audience size) genres: Classic Rock/Oldies and Country. This is consistent with the notion that this form of traditional univorousness stands at maximum sociodemographic contrast in relation to middle-class eclecticism (Holt, 1998; Warde et al., 2008). 


\section{Discussion and Conclusion}

\subsection{Implications for our understanding of taste and genre dynamics}

In terms of our understanding of the dynamic of genres and cultural choices, the analysis presented in this paper reveals a number of insights, both theoretical and substantive. First, in terms of theory, the approach developed in the foregoing shows that many of the workhorse metrics in the empirical study of cultural taste, such as "omnivorousness by volume" or genre popularity are already incipient relational metrics because they technically rank persons and cultural objects based on their pattern of connectivity within a (usually implicit) two-mode cultural network. The reflective approach builds on this foundation and takes this a step further, revealing a number of additional relational metrics that seem to operate according to a similar logic.

In this last respect, there is reason to suspect that this is more than a modeling or measurement exercise, as all of these metrics may be getting at something substantively fundamental about the way that people make cultural choices. The idea here is that precisely because genres "take up" the relational properties of their audiences, these properties then reflect back upon persons when they select those objects. These new-fangled properties of persons then become perceptually and motivationally relevant properties of the genres themselves which are taken into consideration when people make cultural choices because persons now come to (mis)perceive them as (substantive) properties of genres (Bryson, 1996; Lizardo, 2006).

The dynamic whereby relational properties of people and things acquire social currency when they are perceived as substantial (e.g. intrinsic) properties is a fundamental tenet of field-theoretic understandings of the relationship between perception and action in general (Martin, 2011), and cultural choice in particular (Bourdieu, 1984). This theoretical insight is fully redeemed and extended in the framework proposed here. The case of popularity is the classic (and simplest) case of this dynamic (Lieberson, 2000). As noted earlier (see equation 2), popularity is a formal property of the genre (the centrality of the genre in the cultural network) which can come to be perceived as substantive property of the object 
("popular culture") which can then itself become a criterion for consumption (or abstention): Persons take-up (as in standard threshold diffusion models) a given cultural form because it is popular. In the same way, as noted in Simmel's (1957) classic statement on fashion, the same relational property can be used as a criterion for rejection on the part of an avantgarde, while its obverse (lack of popularity) can be used as signal that a given form is worth engaging.

The same goes for the way in which formal properties of cultural forms "reflect back" on persons when they "associate" with those forms: A person with a high taste for popularity (and average of the first-order relational properties of the genres chosen) can be perceived as a less than discerning dabbler in "common" culture and be rejected (or embraced) on precisely that basis (Bourdieu, 1984). In this case cultural networks function as reflective "prisms" (Podolny, 2001). A person with a low TFP, on the other hand, can, under some institutional conditions, be seen as connoisseur of hard to access niche culture and thus garner symbolic and social capital on that basis (Arsel and Thompson, 2011).

In addition, the relational properties of cultural forms can be relevant for culture consumption outcomes not only based on what those properties mean to persons (e.g. in terms of their first order preferences) but also in terms of what those properties signal to others about the person (third order beliefs). For instance, Obukhova et al. (2014) show that in a socio-political context in which a taste for popularity signals loyalty to interested third parties, and persons have a strong incentive to signal loyalty to others, then persons will be more likely to select popular forms (e.g. first names).

The method of reflections suggests that the same mechanism may be at play in higher order relational properties of both persons and cultural objects. Thus, genres can come to acquire as second order relational properties, the first-order relational properties of their audiences (such as their levels of omnivorousness), and the same goes for persons. As such, genres selected by those who also select a large number of genres may indirectly acquire cachet and status as the sort of culture consumed by those who may want to signal aesthetic cosmopolitanism (Ollivier, 2008). This may be the case for the contemporary status of Hip Hop as an emerging form of cultural capital among middle class white elites (Khan, 2010, 
192). In the same way, genres whose audiences are primarily composed of those who seek out other popular genres may come to acquire the same perceptual trappings (or advantages as the case may be) as those genres, in partial decoupling from their actual levels of popularity.

Naturally, this argument cannot be extended indefinitely, as it violates both intuition and well-established findings on the limits of working memory to suggest that people could keep track of endlessly iterated relational properties of cultural forms (Cowan, 2010). As such, it is not my suggestion that the rank of a genre at higher level of reflections enters directly into the cultural choice process. Instead, after a given limit is reached, the logic of intended consequences stops and that of the unintended consequences or effects takes over.

In this way, lower order relational properties (e.g. popularity, average audience omnivorousness) can enter as the input into fast and cheap heuristics governing choice which then lead people to choosing genres that have higher-order relational properties that they could not have known about ex ante. Note that this argument is meant to be exactly parallel to that explaining the way in which people can make relational choices that garner indirect social capital (the theoretical motivation for the family of eigenvector-style conceptions of centrality). Thus, while it is unlikely that people will know the number of friends that their friends of friends have, they are surprisingly good at using heuristics to "perceive" a person's popularity directly as a substantive property, and thus make a friendship choice on that basis (Waller, 1937).

However, because relational properties tend to be correlated in real-networks (e.g. popular people tend to be attracted to other popular people generating positive degree centrality correlations (Newman, 2002)), a person who chooses a popular person as a contact is also ipso facto making a choice based on a second order property of the person (they are selected as a friend someone who is also friends with popular people) without necessarily using that fact as an input into the choice process (although this information could be visible in some settings, in which case the unintended consequence moves to the third level; choosing a friend whose friends' friends are themselves popular). The same argument would apply to fourth and higher order properties of both other people, and cultural forms. Of course the extent to which higher order heuristics (beyond the classic case of popularity) enter directly 
into people's culture consumption choices is a topic that is ripe for future research.

\subsection{Implications for our understanding of individual patterns of cultural choice}

In terms of our understanding of the link between patterns of cultural choice and positional characteristics at the level of individuals, the analysis reveals that there is meaningful and sociologically relevant information encoded in the seemingly complex patterns of individual trajectory across both the omnivore choice and popular choice bias space of reflective centralities. Partitioning persons into discrete classes based on their trajectories reveals systematic patterns of opposition based on both social position and taste. This includes the capacity to isolate patterns of cultural choice that have both generational and class-based components, from those that are driven by purely-generational (class-orthogonal) dynamics (Lizardo and Skiles, 2015).

While these results are intuitive and cohere with previous work using traditional methods, the reflective centrality approach reveals that we can arrive at them without engaging in the usual (circular) approach of reading taste orientations from the substantive nature of cultural choice patterns (e.g. clustering persons based on the content of their selections). Instead, we can exploit information embedded in the indirect connectivity of genre choices to show that social groups may also garner distinction from the form not just the content of their aesthetic choices (Pachucki and Breiger, 2010; Simmel, 1950). As noted earlier, substantive partitions between genres (e.g. the distinction between legitimate, "authorized" genres, less legitimated offerings (Warde et al., 2008)) then can be seen as a partial outcome of the relational properties that genres acquire as part of their social and institutional trajectory. 


\section{References}

Arsel, Z., Thompson, C. J., 2011. Demythologizing consumption practices: How consumers protect their field-dependent identity investments from devaluing marketplace myths. Journal of Consumer Research 37 (5), 791-806.

Bonacich, P., 1991. Simultaneous group and individual centralities. Social networks 13 (2), 155-168.

Borgatti, S. P., Everett, M. G., 1997. Network analysis of 2-mode data. Social networks 19 (3), $243-269$.

Bourdieu, P., 1984. Distinction: A social critique of the judgment of taste. Harvard University Press, Cambridge, MA.

Boutyline, A., 2017. Improving the measurement of shared cultural schemas with correlational class analysis: Theory and method. Sociological Science 4 (15), 353-393.

Boutyline, A., Vaisey, S., 2017. Belief network analysis: A relational approach to understanding the structure of attitudes. American Journal of Sociology 122 (5), 1371-1447.

Breiger, R. L., 1974. The duality of persons and groups. Social forces 53 (2), 181-190.

Bryson, B., 1996. Anything but heavy metal": Symbolic exclusion and musical dislikes. American sociological review, 884-899.

Bryson, B., 1997. What about the univores? musical dislikes and group-based identity construction among americans with low levels of education. Poetics 25 (2), 141-156.

Cowan, N., 2010. The magical mystery four: How is working memory capacity limited, and why? Current directions in psychological science 19 (1), 51-57.

DiMaggio, P., 1987. Classification in art. American sociological review 52, 440-455.

Faust, K., 1997. Centrality in affiliation networks. Social networks 19 (2), 157-191.

Fishman, R. M., Lizardo, O., 2013. How macro-historical change shapes cultural taste legacies of democratization in spain and portugal. American Sociological Review 78 (2), 213-239.

Freeman, L. C., 1978. Centrality in social networks conceptual clarification. Social networks 1 (3), $215-239$.

Gans, H., 1999. Popular culture and high culture: An analysis and evaluation of taste. Basic Books.

Goldberg, A., 2011. Mapping shared understandings using relational class analysis: The case of the cultural omnivore reexamined. American Journal of Sociology 116 (5), 1397-1436.

Goldberg, A., Hannan, M. T., Kovács, B., 2016. What does it mean to span cultural boundaries? variety and atypicality in cultural consumption. American Sociological Review, 0003122416632787.

Hidalgo, C. A., Hausmann, R., 2009. The building blocks of economic complexity. proceedings of the national academy of sciences 106 (26), 10570-10575.

Holt, D. B., 1998. Does cultural capital structure american consumption? Journal of consumer research $25(1), 1-25$.

Jung, T., Wickrama, K., 2008. An introduction to latent class growth analysis and growth mixture modeling. 
Social and Personality Psychology Compass 2 (1), 302-317.

Khan, S. R., 2010. Privilege: The making of an adolescent elite at St. Paul's School. Princeton University Press, Princeton, NJ.

Kovács, B., 2010. A generalized model of relational similarity. Social Networks 32 (3), 197-211.

Lewis, K., Gonzalez, M., Kaufman, J., 2012. Social selection and peer influence in an online social network. Proceedings of the National Academy of Sciences 109 (1), 68-72.

Lieberson, S., 2000. A matter of taste: How names, fashions, and culture change. Yale University Press, New Haven.

Lizardo, O., 2006. How cultural tastes shape personal networks. American Sociological Review 71 (5), $778-$ 807.

Lizardo, O., 2008. The question of culture consumption and stratification revisited. Sociologica 2 (2), 0-0.

Lizardo, O., 2014. Omnivorousness as the bridging of cultural holes: A measurement strategy. Theory and Society 43 (3-4), 395-419.

Lizardo, O., Skiles, S., 2015. Musical taste and patterns of symbolic exclusion in the united states 1993-2012: Generational dynamics of differentiation and continuity. Poetics 53, 9-21.

Lizardo, O., Skiles, S., 2016a. Cultural objects as prisms perceived audience composition of musical genres as a resource for symbolic exclusion. Socius: Sociological Research for a Dynamic World 2, 2378023116641695

Lizardo, O., Skiles, S., 2016b. The end of symbolic exclusion? the rise of categorical tolerance in the musical tastes of americans: 1993-2012. Sociological Science 3, 85-108.

Mark, N. P., 2003. Culture and competition: Homophily and distancing explanations for cultural niches. American sociological review 68, 319-345.

Martin, J. L., 2011. The explanation of social action. Oxford University Press, New York.

Maslov, S., Sneppen, K., Zaliznyak, A., 2004. Detection of topological patterns in complex networks: correlation profile of the internet. Physica A: Statistical Mechanics and its Applications 333, 529-540.

Mealy, P., Doyne Farmer, J., Teytelboym, A., 2018. A new interpretation of the economic complexity index. arXiv preprint arXiv:1711.08245.

URL https://arxiv.org/abs/1711.08245

Morrison, G., Buldyrev, S. V., Imbruno, M., Arrieta, O. A. D., Rungi, A., Riccaboni, M., Pammolli, F., 2017. On economic complexity and the fitness of nations. Scientific Reports 7 (1), 15332.

Newman, M. E., 2002. Assortative mixing in networks. Physical review letters 89 (20), 208701.

Newman, M. E., 2006. Finding community structure in networks using the eigenvectors of matrices. Physical review E 74 (3), 036104.

Obukhova, E., Zuckerman, E. W., Zhang, J., 2014. When politics froze fashion: The effect of the cultural 
revolution on naming in beijing. American Journal of Sociology 120 (2), 555-583.

Ollivier, M., 2008. Modes of openness to cultural diversity: Humanist, populist, practical, and indifferent. poetics 36 (2), 120-147.

Pachucki, M. A., Breiger, R. L., 2010. Cultural holes: Beyond relationality in social networks and culture. Annual review of sociology 36, 205-224.

Peterson, R. A., 1983. Patterns of cultural choice a prolegomenon. American Behavioral Scientist 26 (4), $422-438$

Peterson, R. A., 1992. Understanding audience segmentation: From elite and mass to omnivore and univore. Poetics 21 (4), 243-258.

Peterson, R. A., Kern, R. M., 1996. Changing highbrow taste: From snob to omnivore. American sociological review, 900-907.

Podolny, J. M., 2001. Networks as the pipes and prisms of the market 1. American journal of sociology 107 (1), 33-60.

Silver, D., Lee, M., Childress, C. C., 2016. Genre complexes in popular music. PloS one 11 (5), e0155471.

Simmel, G., 1950. The sociology of georg simmel. Simon \& Schuster.

Simmel, G., 1957. Fashion. American journal of sociology 62 (6), 541-558.

Sonnett, J., 2016. Ambivalence, indifference, distinction: A comparative netfield analysis of implicit musical boundaries. Poetics 54, 38-53.

Vaisey, S., Lizardo, O., 2010. Can cultural worldviews influence network composition? Social Forces 88 (4), $1595-1618$.

Vlegels, J., Lievens, J., 2017. Music classification, genres, and taste patterns: A ground-up network analysis on the clustering of artist preferences. Poetics 60, 76-89.

Waller, W., 1937. The rating and dating complex. American sociological review 2 (5), 727-734.

Warde, A., Wright, D., Gayo-Cal, M., 2008. The omnivorous orientation in the uk. Poetics 36 (2), 148-165. 


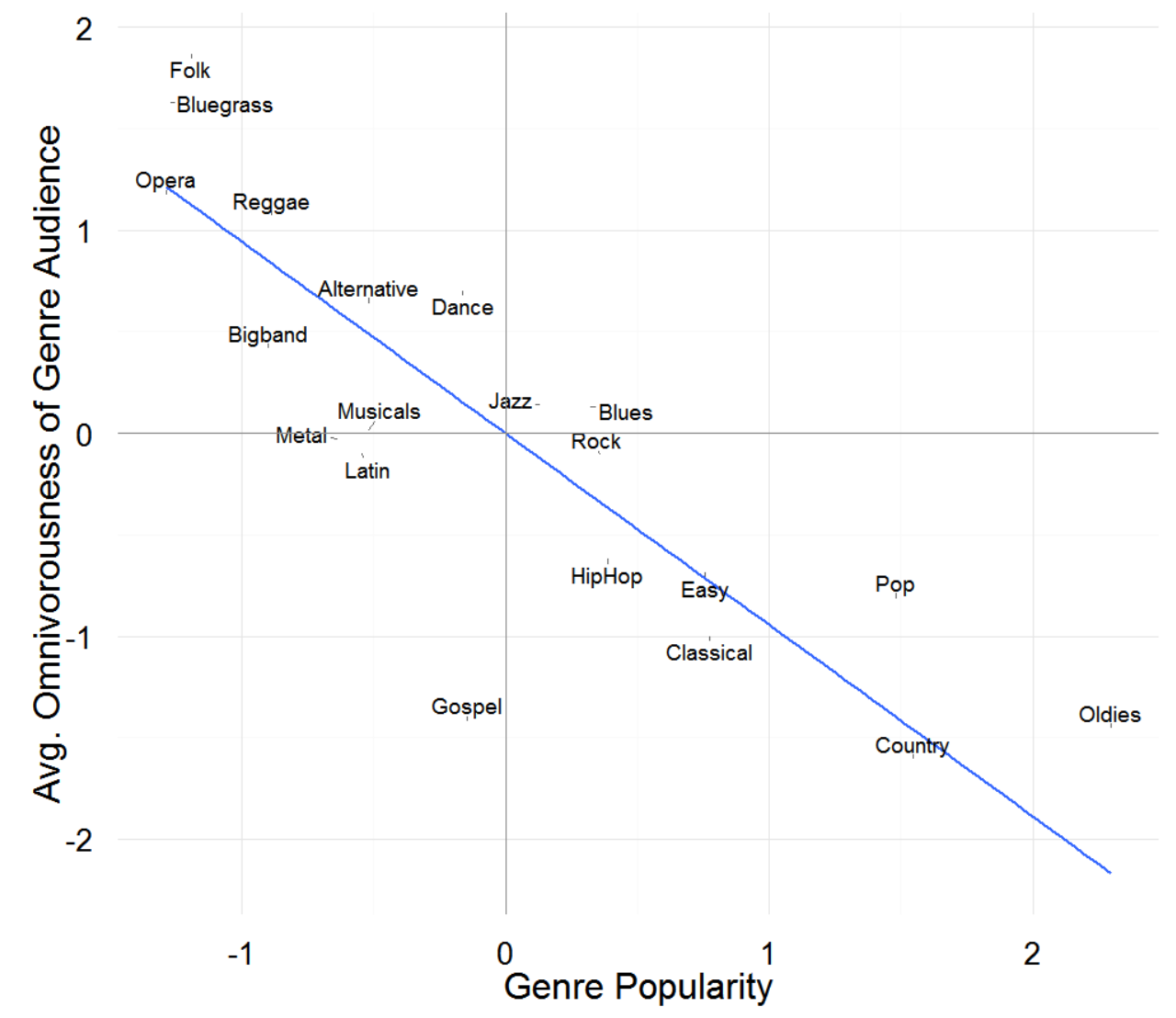

(a) Plot of observed genre popularity $\left(\delta_{k 0}\right)$ against genre omnivorousness $\left(\delta_{k 1}\right)$. Regression line corresponds to expected correlation of the two quantities in reshuffled data.
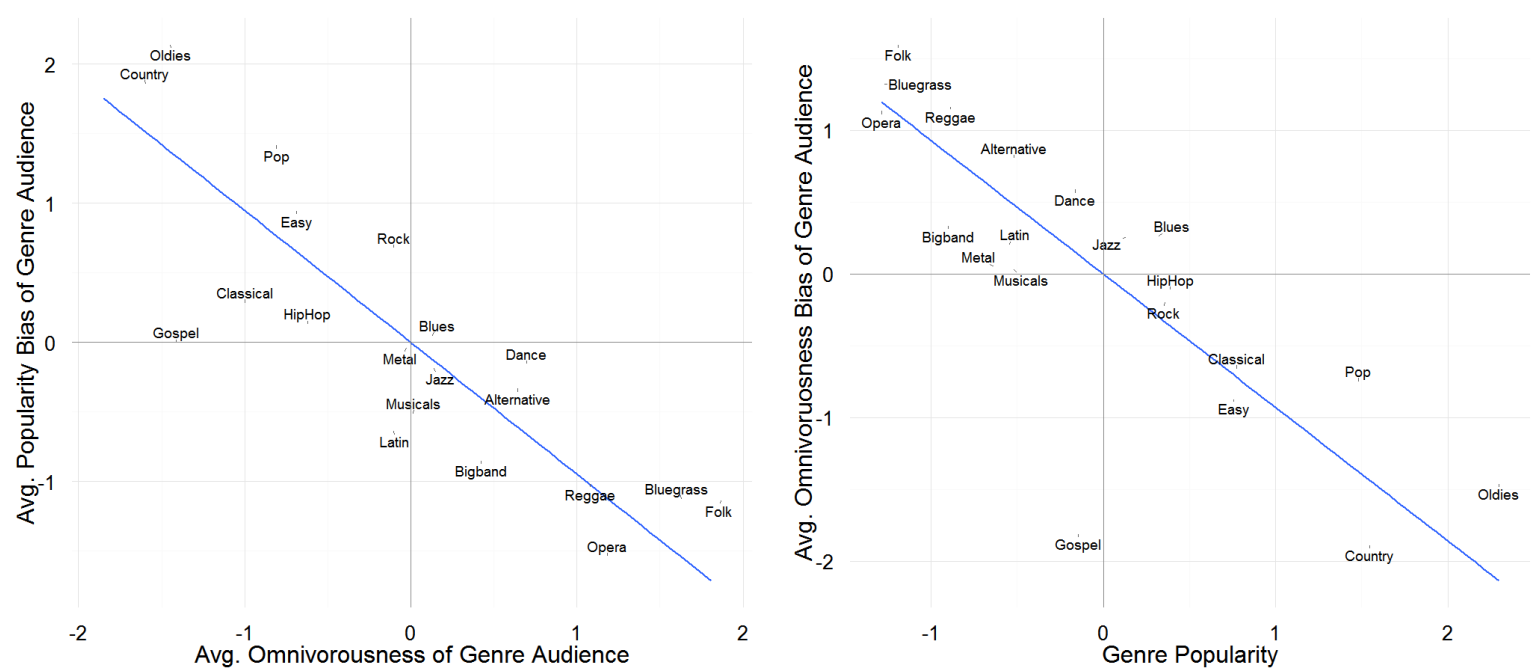

(b) Plot of genre omnivorousness $\left(\delta_{k 1}\right)$ against genre popularity bias $(\mathrm{c})$ Plot of genre popularity $\left(\delta_{k 0}\right)$ against genre omnivorousness bias $\left(\delta_{k 2}\right)$. Regression line corresponds to expected correlation of the two $\left(\delta_{k 3}\right)$. Regression line corresponds to expected correlation of the two quantities in reshuffled data. quantities in reshuffled data.

Figure 3 


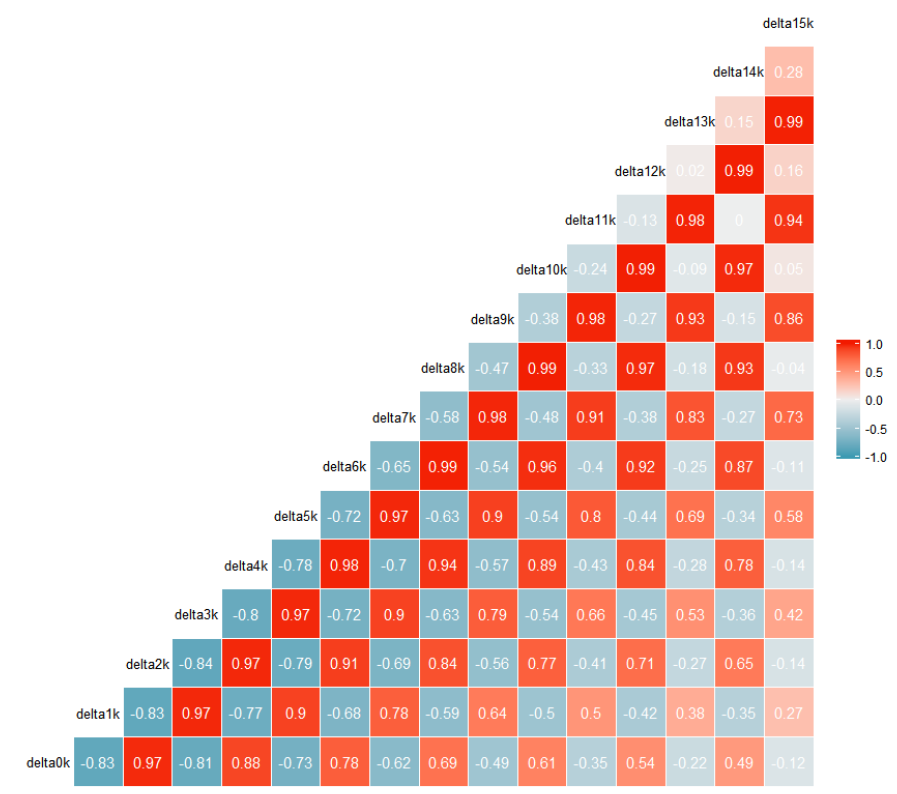

(a) Correlations in Original Data

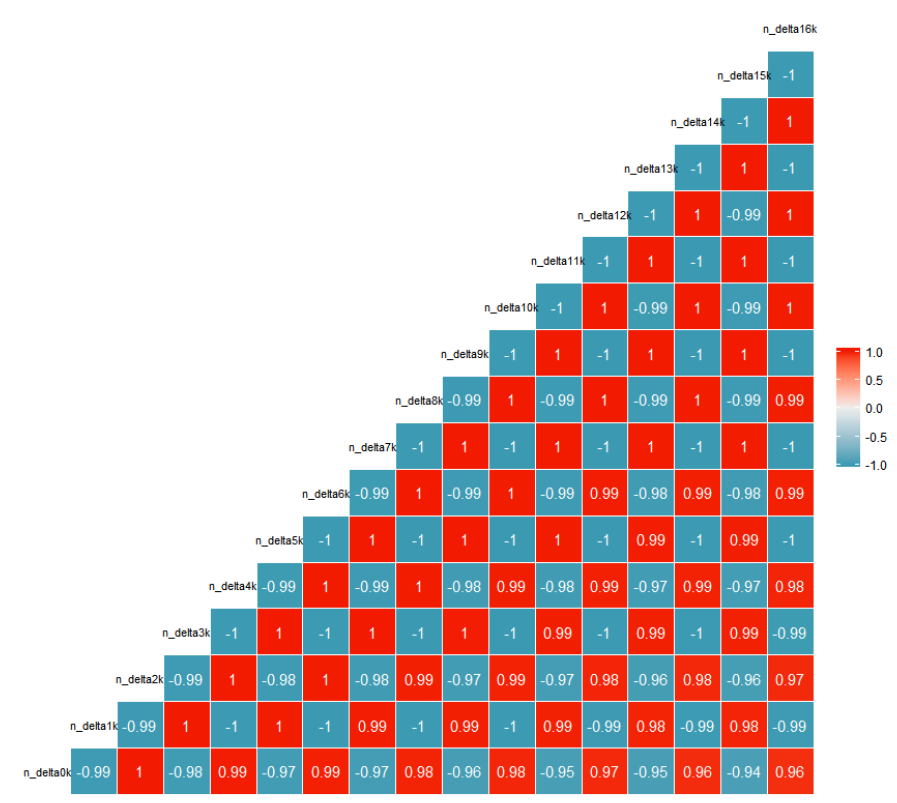

(b) Correlations in Reshuffled Data

Figure 4 


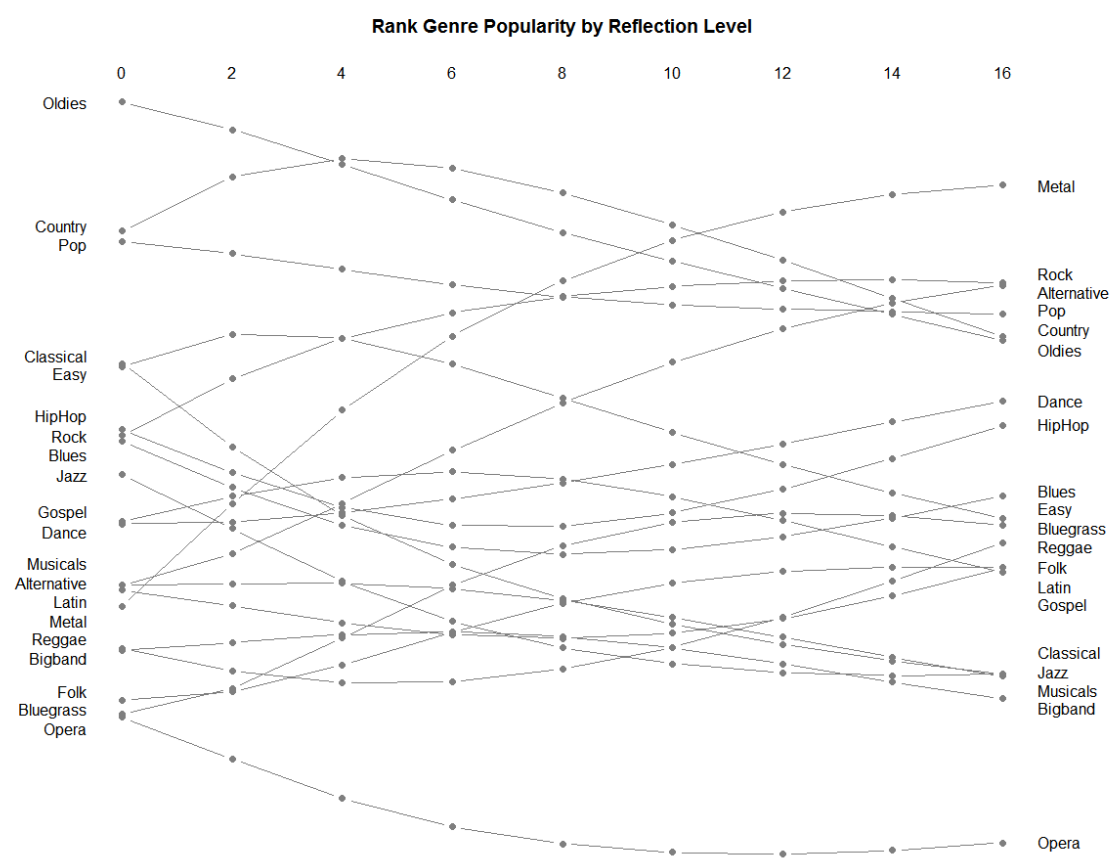

(a) Genre centrality trajectories in original data

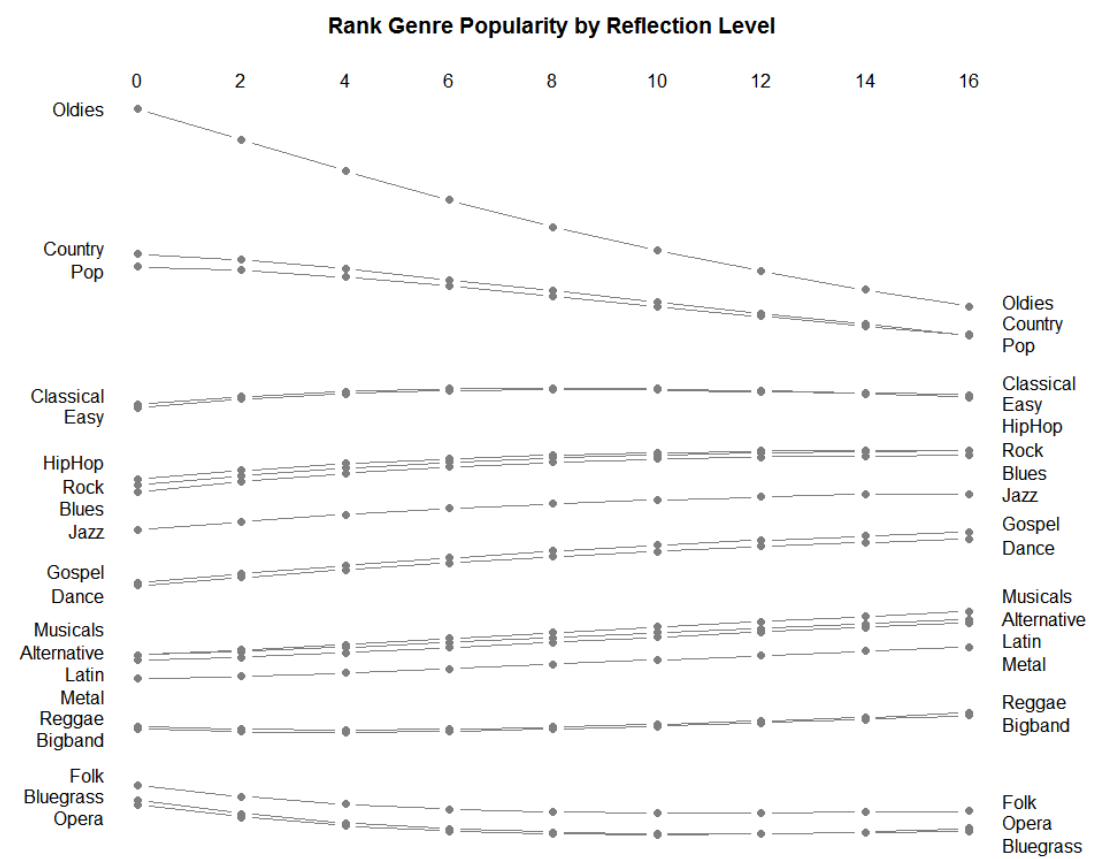

(b) Genre centrality trajectories in reshuffled data

Figure 5: Slopegraphs of reflective genre popularity, $M=16$ 


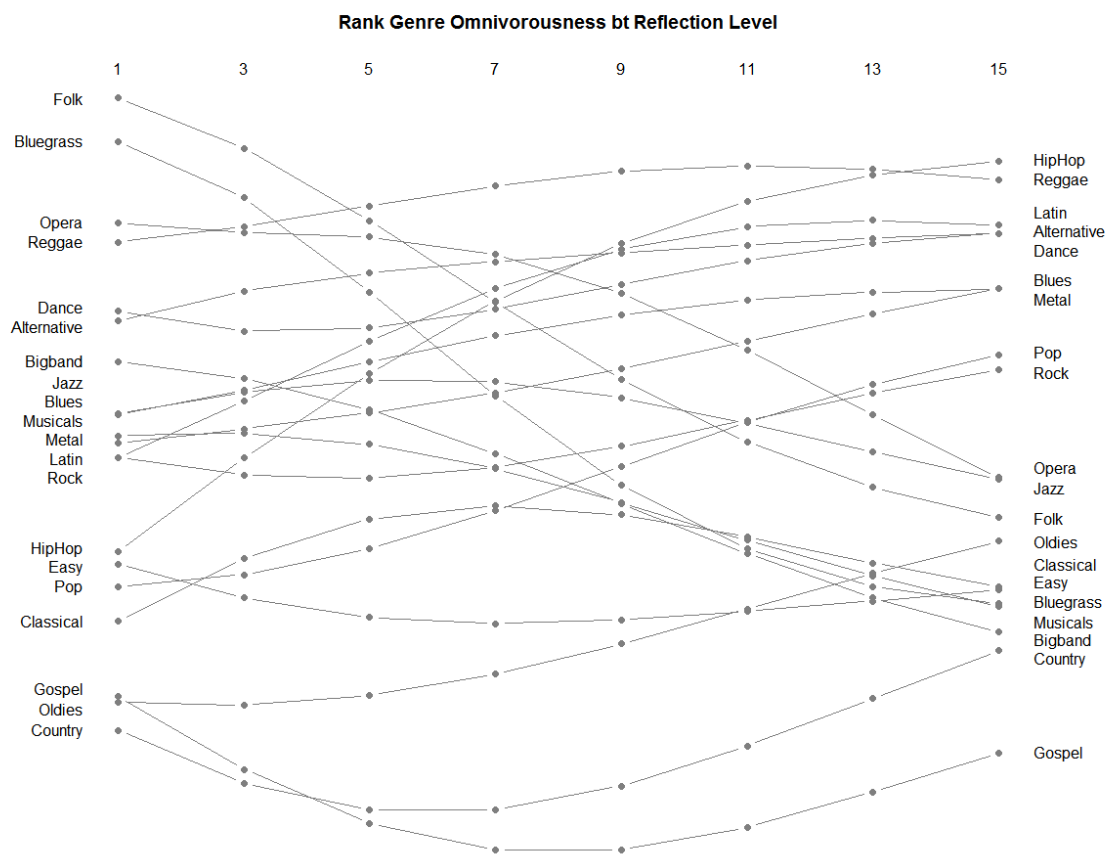

(a) Genre centrality trajectories in original data

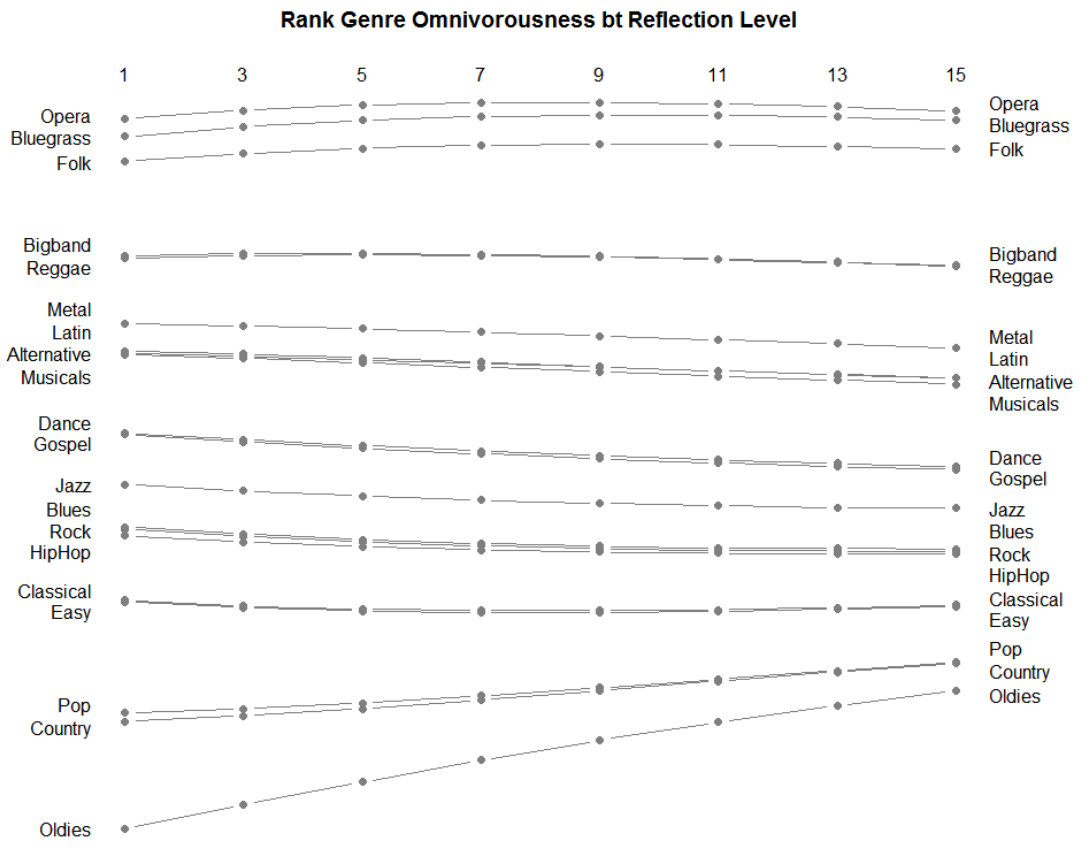

(b) Genre centrality trajectories in reshuffled data

Figure 6: Slopegraphs of reflective genre omnivorousness, $M=16$ 


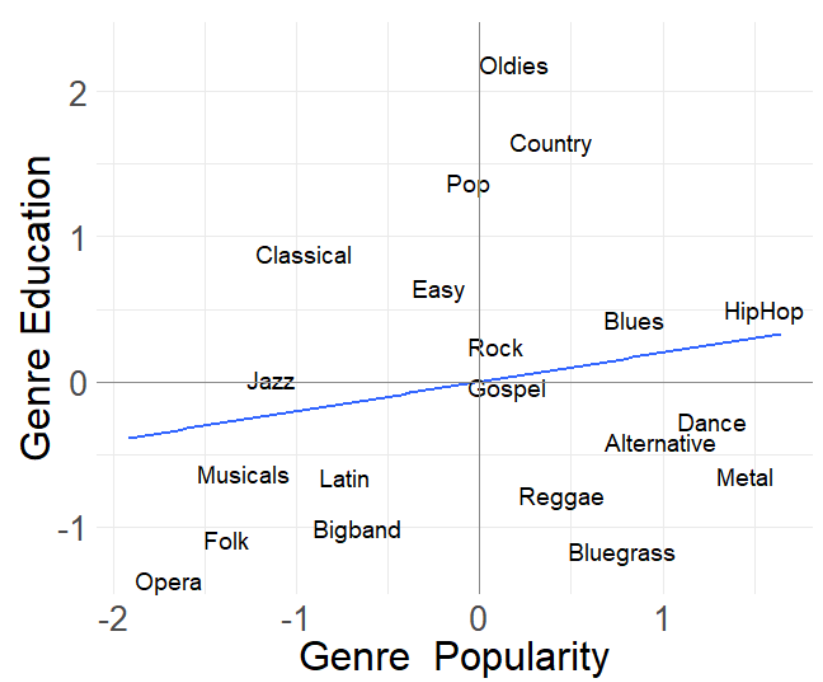

(a) Genre Educ. (Standardized) by Popularity (Standardized).

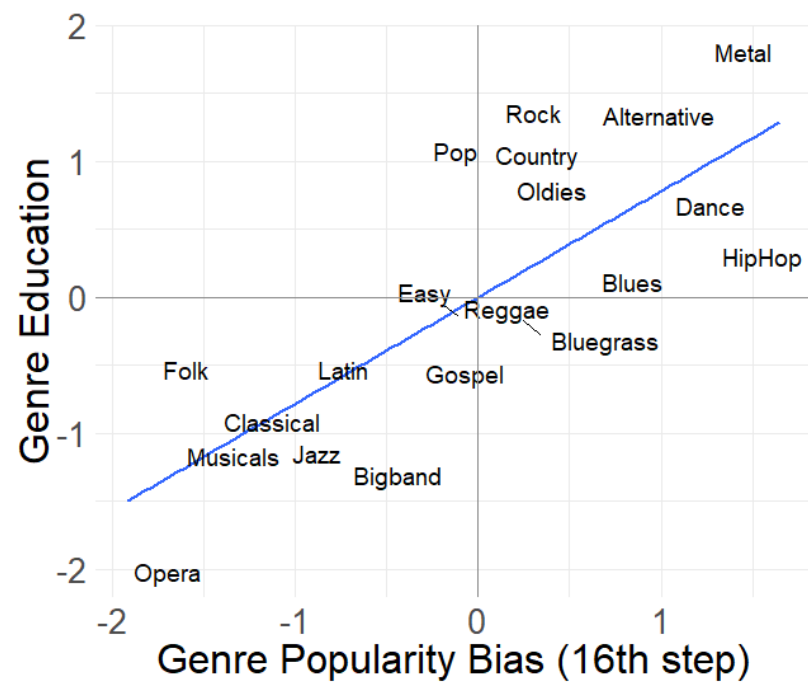

(b) Genre Educ. (Standardized) by Popularity Bias (16 th step) (Standardized)
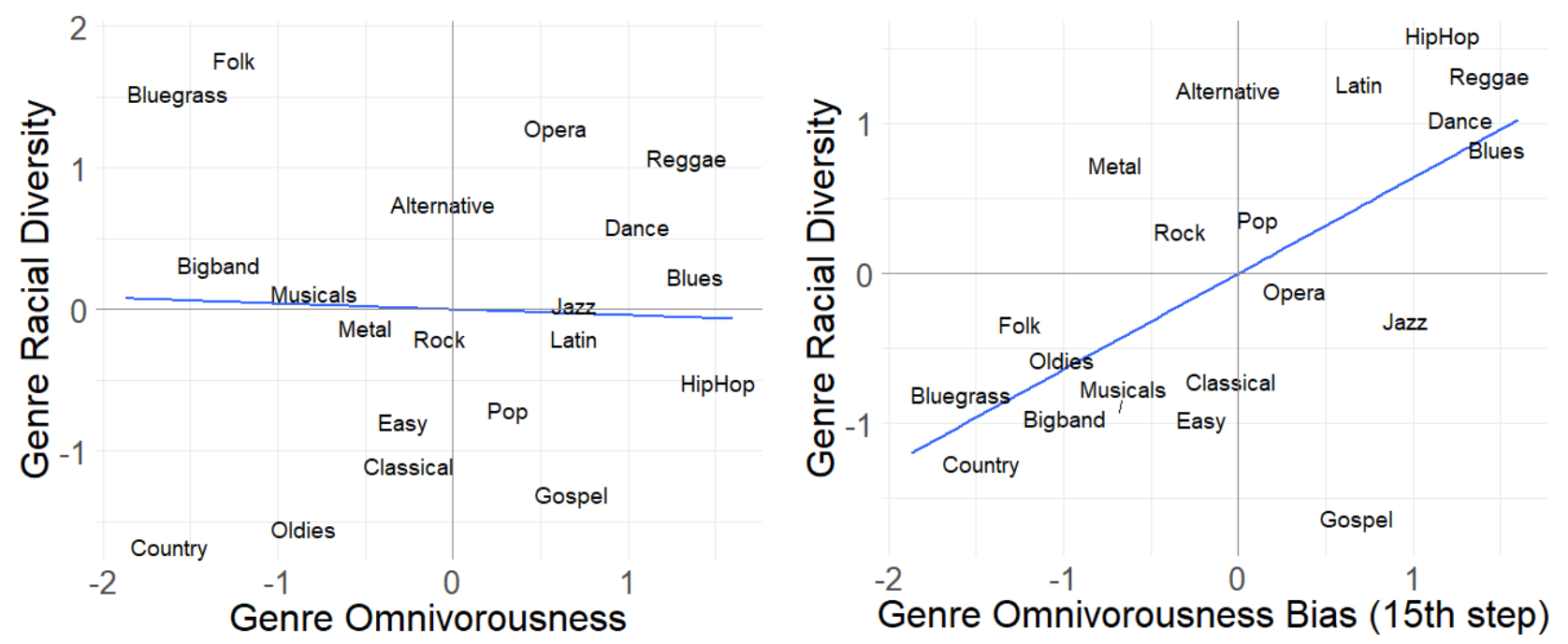

(c) Genre Racial Div. (Standardized) by Genre Omnivorousness (Stan-(d) Racial Div. (Standardized) by Omnivorousness Bias (15 ${ }^{\text {th }}$ step) (Standardized) dardized)

Figure 7 


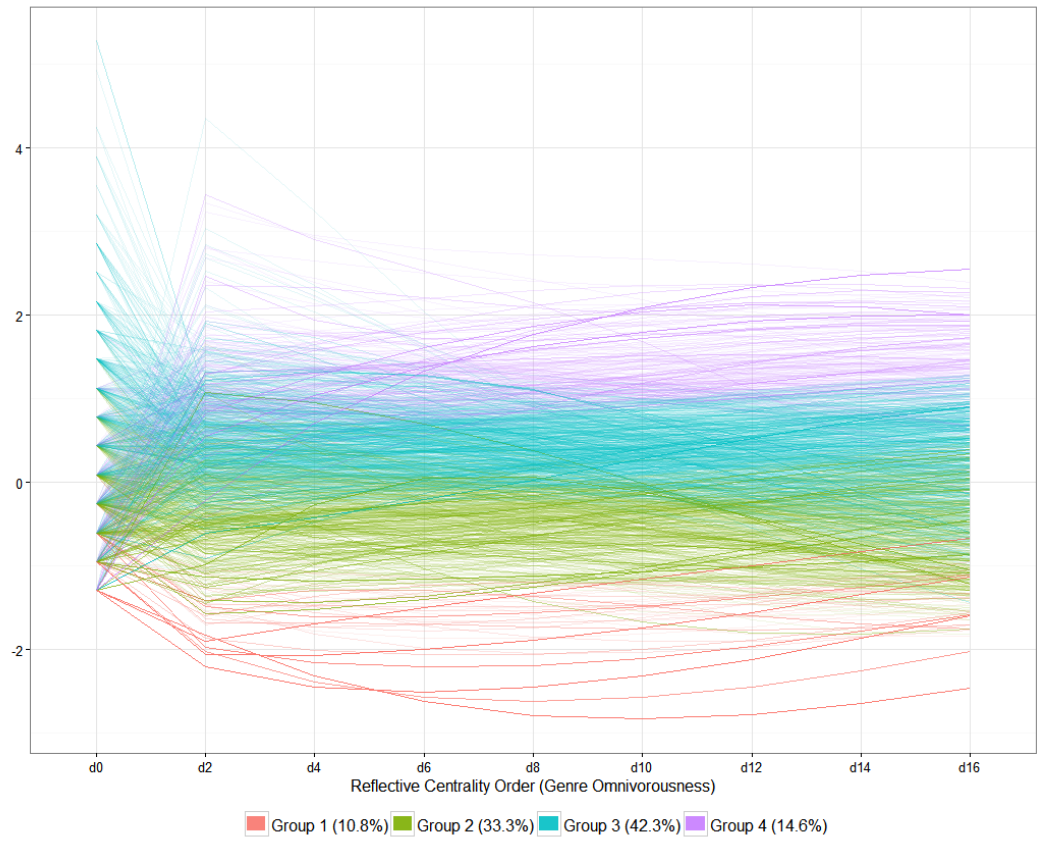

(a) Individual centrality trajectories (Popularity)

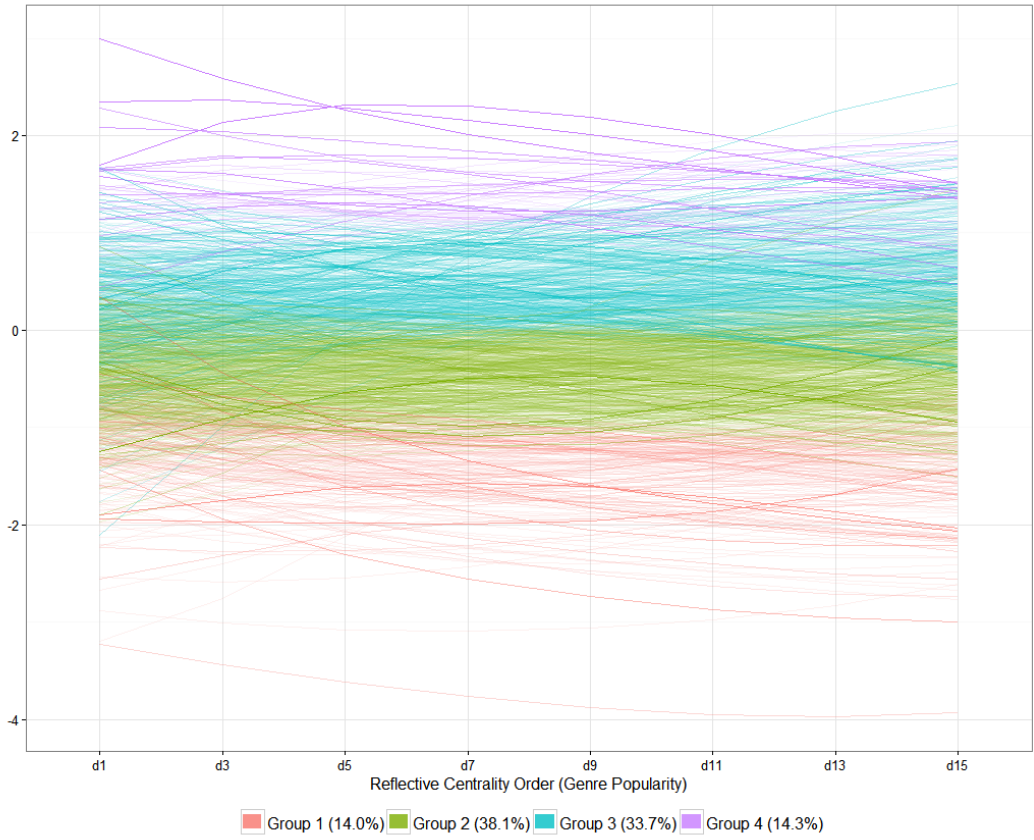

(b) Individual centrality trajectories (Omnivorousness)

Figure 8 


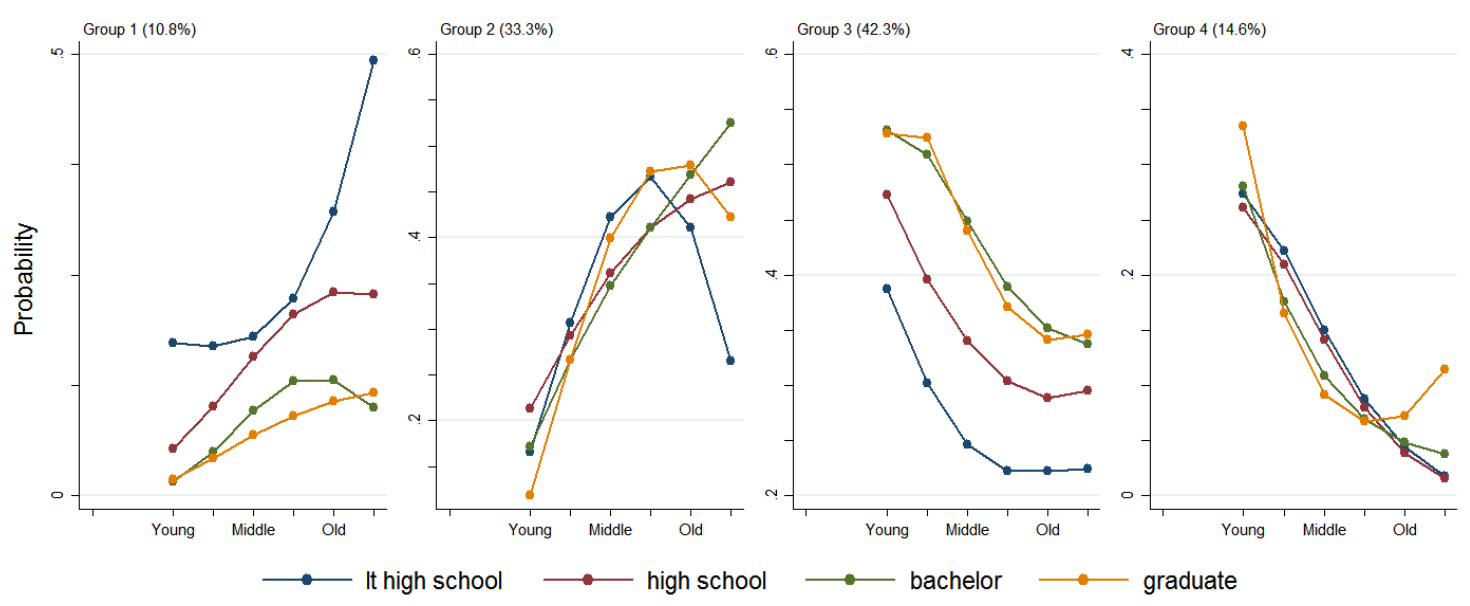

Genre Omnivorousness Centraliity Trajectory Membership Predictors

(a)

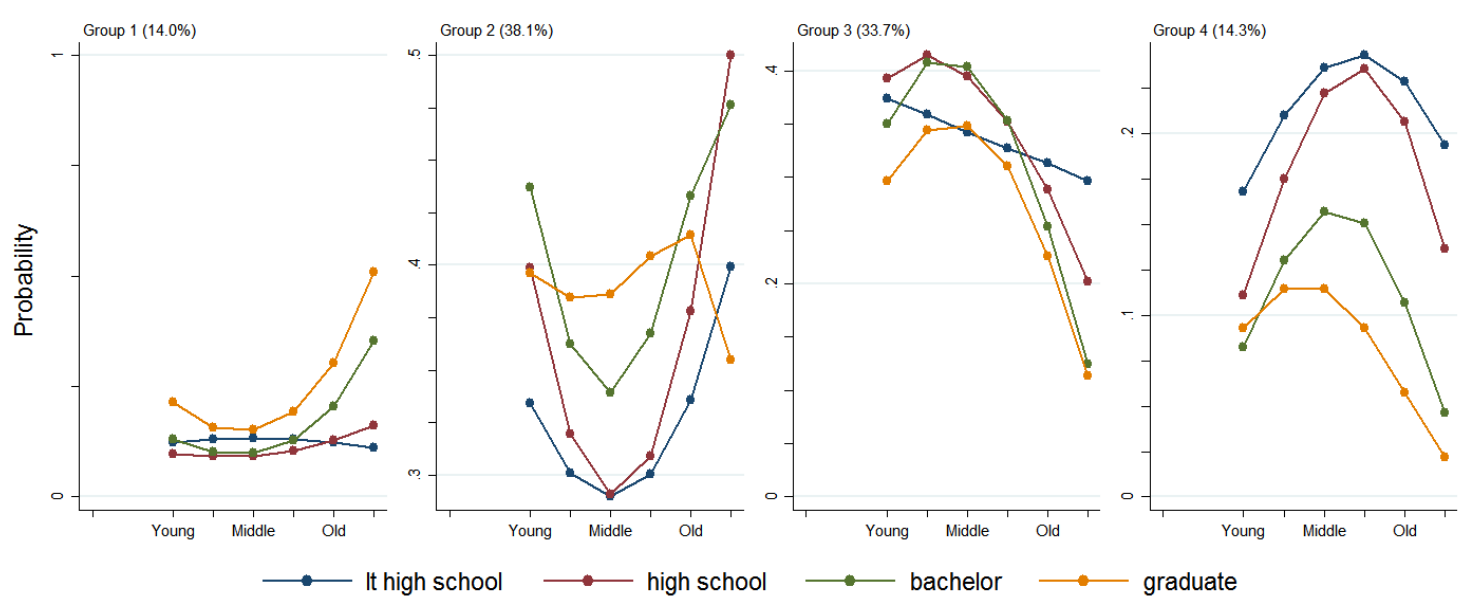

Genre Popularity Centraliity Trajectory Membership Predictors

(b)

Figure 9 


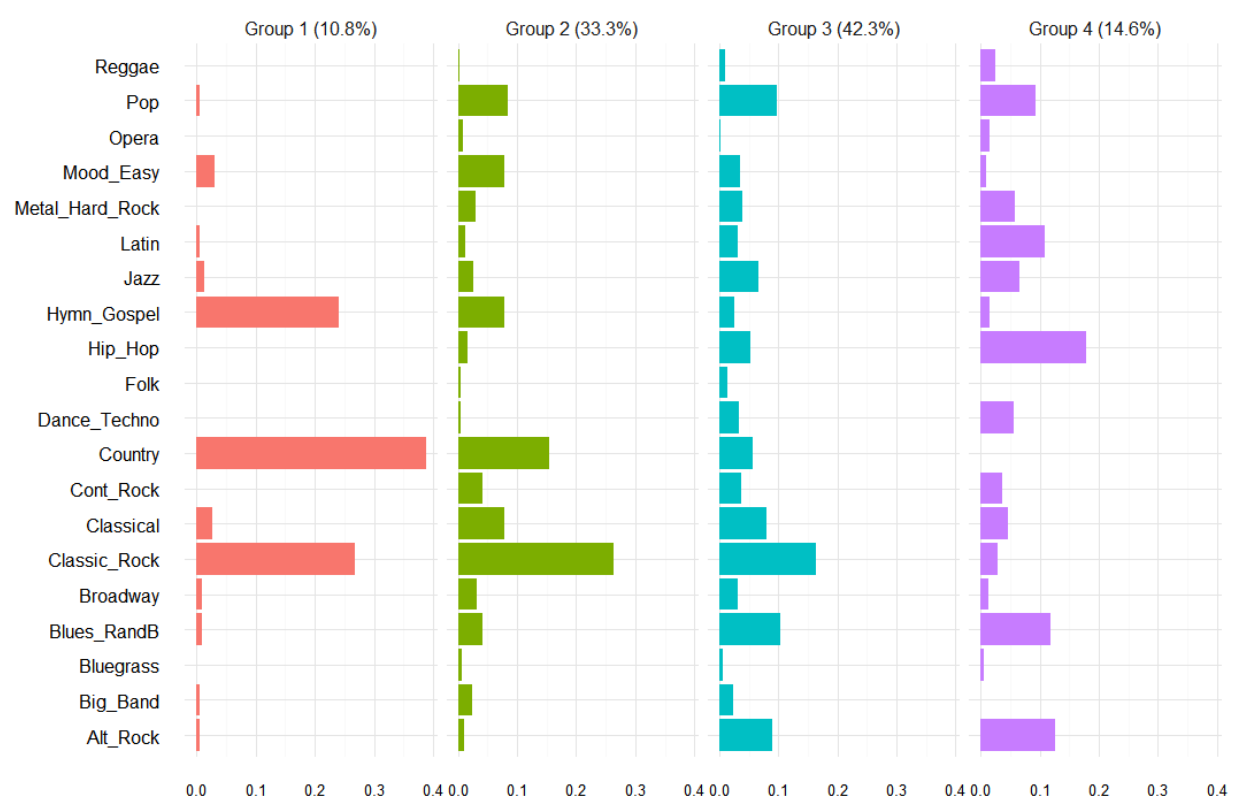

Group 1 (10.8\%) $\square$ Group 2 (33.3\%) $\square$ Group $3(42.3 \%) \square$ Group 4 (14.6\%)

(a) Favorite Musical Genres by Trajectory Class (Omnivore Choice Bias Centralities)

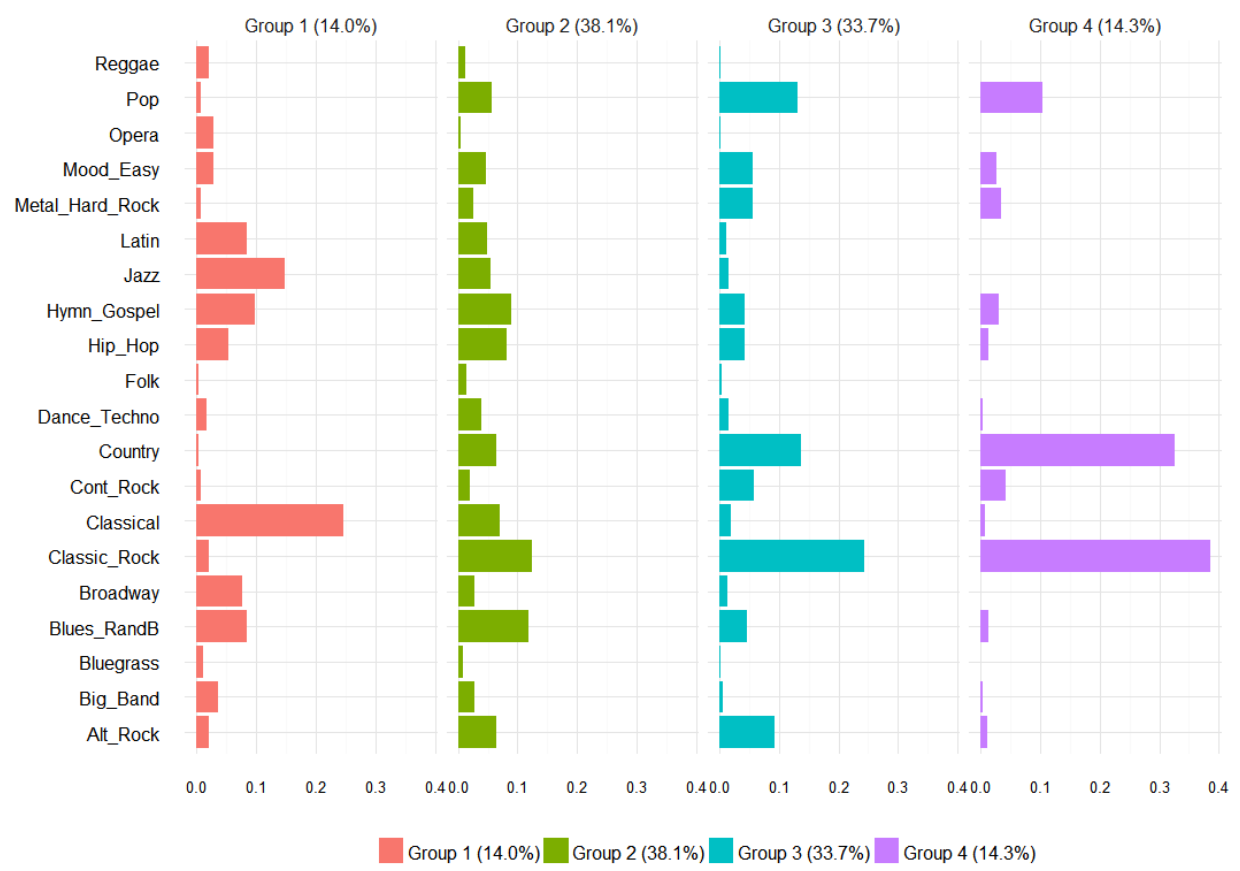

(b) Favorite Musical Genres by Trajectory Class (Popular Choice Bias Centralities)

Figure 10 